\title{
Averaging residue currents and the Stückrad-Vogel algorithm
}

Alain Yger

\begin{abstract}
Trace formulas (Lagrange, Jacobi-Kronecker, Bergman-Weil) play a key role in division problems in analytic or algebraic geometry (including arithmetic aspects, see for example [10]). Unfortunately, they usually hold within the restricted frame of complete intersections. Besides the fact that it allows to carry local or semi global analytic problems to a global geometric setting (think about Crofton's formula), averaging the Cauchy kernel (from $\mathbb{C}^{n} \backslash\left\{z_{1} \ldots z_{n}=0\right\} \subset \mathbb{P}^{n}(\mathbb{C})$ ), in order to get the Bochner-Martinelli kernel (in $\mathbb{C}^{n+1} \backslash\{0\} \subset \mathbb{P}^{n+1}(\mathbb{C})=\mathbb{C}^{n+1} \cup \mathbb{P}^{n}(\mathbb{C})$ ), leads to the construction of explicit candidates for the realization of Grothendieck's duality, namely BM residue currents $([27,3,6])$, extending thus the cohomological incarnation of duality which appears in the complete intersection or Cohen-Macaulay cases. We will recall here such constructions and, in parallel, suggest how far one could take advantage of the multiplicative inductive construction introduced in [13] by N. Coleff and M. Herrera, by relating it to the Stückrad-Vogel algorithm developed in ([30],[31],[8]) towards improper intersection theory. Results presented here were initiated all along my long term collaboration with Carlos Berenstein. To both of us, the mathematical work of Leon Ehrenpreis certainly remained a constant and how much stimulating source of inspiration. This presentation relies also deeply on my collaboration with M. Andersson, H. Samuelsson and E. Wulcan in Göteborg, through the past years.
\end{abstract}

\section{Coleff-Herrera residue currents for complete intersections and the Transformation Law}

Let $\mathcal{X}$ be a $n$-dimensional (ambient) complex manifold and $V \subset \mathcal{X}$ be a closed analytic subset with pure codimension $M$, equipped with its structure

Alain Yger, Institut de Mathématiques, Université de Bordeaux, 33405, Talence, France, e-mail: Alain.Yger@math.u-bordeaux1.fr 
sheaf $\mathcal{O}_{V}=\mathcal{O}_{\mathcal{X}} / \mathcal{I}_{V}$, where $\mathcal{I}_{V, x}=\left\{h_{x} \in \mathcal{O}_{\mathcal{X}, x} ; h_{x}=0\right.$ on $\left.V_{x}\right\}$. Let $[V]$ be the integration current on the complex subspace $\left(V, \mathcal{O}_{V}\right)$. Let $\Delta_{1}, \ldots, \Delta_{m}$ be $m \leq n-M$ Cartier divisors on $\mathcal{X}$, with respective holomorphic global sections $s_{1}, \ldots, s_{m}$, such that for all $x \in V \cap \bigcup_{j=1}^{m} s_{j}^{-1}(0)$, the germs $\left(s_{1, x}, \ldots, s_{m, x}\right)$ define a regular sequence in $\mathcal{O}_{V, x}$. We denote as $\mathcal{O}_{V}[s]$ the ideal sheaf of $\mathcal{O}_{V}$ obtained as the image of $\bigoplus_{j=1}^{m} \mathcal{O}_{V}\left(-\Delta_{j}\right)$ by the interior product with $\left(s_{1}, \ldots, s_{m}\right)$. Note that, if the closed hypersurfaces $s_{j}^{-1}(0), j=1, \ldots, m$, intersect properly on $V$ (that is $\operatorname{dim}\left(V \cap \bigcap_{j=1}^{m} s_{j}^{-1}(0)\right) \leq n-M-m$ ) or, which is equivalent, define a complete intersection on $V$, the required condition holds provided that the ambient manifold $\mathcal{X}$ is replaced by some convenient neighborhood $U$ of $V \cap s^{-1}(0):=V \cap \bigcap_{j=1}^{m} s_{j}^{-1}(0)$. What seems to be today the most robust approach towards the so-called Coleff-Herrera current $\bigwedge_{j=1}^{m} \bar{\partial}\left(1 / s_{j}\right) \wedge[V]$ (originally introduced by N. Coleff and M. Herrera in their pioneer work [13]) is the following result (initially obtained in [9] for $m=2$, then finally extended by H. Samuelsson [28] for arbitrary $m$ ) :

Theorem 1 (robust approach to the Coleff-Herrera residue current attached to a complete intersection). In the above context, for any choice of $C^{\infty}$ metrics ||$_{j}$ on the line bundles $\mathcal{O}_{\mathcal{X}}\left(\Delta_{j}\right)$, the holomorphic $\bigoplus_{r=0}^{m} \bigoplus_{1 \leq j_{1}<\cdots<j_{r} \leq m}{ }^{\prime} \mathcal{D}^{(M, M+r)}\left(\mathcal{X}, \bigwedge_{l=1}^{r} \mathcal{O}_{\mathcal{X}}\left(-\Delta_{j_{l}}\right)\right)$-valued map

$$
\begin{aligned}
& \left(\lambda_{1}, \ldots, \lambda_{m}\right) \in\left\{\lambda ; \operatorname{Re} \lambda_{j}>1, j=1, \ldots, m\right\} \\
& \longmapsto\left[\bigwedge_{j=m}^{1}\left(1-\left|s_{j}\right|_{j}^{2 \lambda_{j}}+\frac{1}{2 i \pi} \bar{\partial}\left(\frac{\left|s_{j}\right|_{j}^{2 \lambda_{j}}}{s_{j}}\right)\right)\right] \wedge[V]
\end{aligned}
$$

can be analytically continued as an holomorphic map to a product of halfspaces $\left\{\operatorname{Re} \lambda_{j}>-\eta\right\}$ for some $\eta>0$. Its value at $\lambda=0$ coincides with its ' $\mathcal{D}^{M, M+r}\left(\mathcal{X}, \bigwedge_{j=1}^{m} \mathcal{O}_{\mathcal{X}}\left(-\Delta_{j}\right)\right)$-component and defines a $\bar{\partial}$-closed bundlevalued current which is independent of the metrics ||$_{j}, j=1, \ldots, m$. This current, supported by $V \cap s^{-1}(0)$, is denoted as $\bigwedge_{j=1}^{m} \bar{\partial}\left(1 / s_{j}\right) \wedge[V]$. Considered as a $\bar{\partial}$-closed current on the complex space $\left(V, \mathcal{O}_{V}\right)$, it is locally annihilated by any local section of the ideal sheaf $\mathcal{O}_{V}[s]$. Moreover, when $V=\mathcal{X}$, it realizes the local duality with respect to the ideal sheaf $\mathcal{O}_{\mathcal{X}}[s]$, namely

$$
\left(h_{x} \cdot \bigwedge_{j=1}^{m} \bar{\partial}\left(1 / s_{j}\right)=0\right) \Longleftrightarrow\left(h_{x} \in\left(\mathcal{O}_{\mathcal{X}}[s]\right)_{x}=\left(s_{1, x}, \ldots, s_{m, x}\right) \mathcal{O}_{\mathcal{X}, x}\right)
$$

The intimate relationship between the sheaf of differential operators with meromorphic coefficients and the local description of such a current arises from the following remark, which refers to the sheaves of ColeffHerrera currents (here bundle-valued, holomorphic or meromorphic, see [32]) introduced by J.E. Björk in [11], together with their companion local structure theorems (see [11], or also [32] for a survey in this volume). The current $\bigwedge_{j=1}^{m} \bar{\partial}\left(1 / s_{j}\right) \wedge[V]$ splits locally about each point of its sup- 
port $V \cap s^{-1}(0)$ as $\mathcal{T}_{x} \wedge \omega_{x}$, where $\mathcal{T}_{x}$ is a local section of the ColeffHerrera sheaf $\mathrm{CH}_{\mathcal{X}, V \cap s^{-1}(0)}\left(\cdot ; \star S_{x}, \bigwedge_{j=1}^{m} \mathcal{O}_{\mathcal{X}}\left(-\Delta_{j}\right)\right)$, and $\omega_{x}$ is a local section of $\Omega_{\mathcal{X}}^{M+m}\left(\cdot, \bigwedge_{1}^{m} \mathcal{O}_{\mathcal{X}}\left(\Delta_{j}\right)\right)$. Here $S_{x}$ denotes a germ of hypersurface at $x$ polar respect to $V_{x} \cap s_{x}^{-1}(0)$, i.e. $\overline{\left(V_{x} \cap s_{x}^{-1}(0)\right) \backslash S_{x}}=V_{x} \cap s_{x}^{-1}(0)$. Moreover, when $V$ is Cohen-Macaulay about $x_{0} \in V \cap s^{-1}(0)$ (that is $\mathcal{O}_{V, x_{0}}$ is Cohen-Macaulay), the integration current $[V]$ factorizes about $x_{0}$ as $\tau_{x_{0}} \wedge \varpi_{x_{0}}$, where $\varpi_{x_{0}}$ is a local section of the Coleff-Herrera sheaf $\mathrm{CH}_{\mathcal{X}, V}$, and $\varpi_{x_{0}}$ is a section of the sheaf $\Omega_{\mathcal{X}}^{M}$ (see [4]), which implies in this particular case that one can take about $x_{0}$ the current section $\mathcal{T}_{x_{0}}$ to be a local section $T_{x_{0}}$ of $\mathrm{CH}_{\mathcal{X}, V \cap s^{-1}(0)}\left(\cdot, \bigwedge_{1}^{m} \mathcal{O}_{\mathcal{X}}\left(-\Delta_{j}\right)\right)$ instead of $\mathrm{CH}_{\mathcal{X}, V \cap s^{-1}(0)}\left(\cdot ; \star S_{x_{0}}, \bigwedge_{1}^{m} \mathcal{O}_{\mathcal{X}}\left(-\Delta_{j}\right)\right)$. This occurs in particular when $V$ is defined as a reduced complete intersection about the point $x_{0}$.

Remark 1. Theorem 1 provides a robust approach (via analytic continuation) towards the Coleff-Herrera residue current for complete intersections on a purely dimensional analytic set $\left(V, \mathcal{O}_{V}\right)$, as developed in [13]. It is important here to point out that one may replace in (1) the integration current $[V]$ by any global section of the Coleff-Herrera sheaf $\mathrm{CH}_{\mathcal{X}, V}(\cdot, E)$, where $E$ denotes a finite rank holomorphic bundle over $\mathcal{X}$. When $m \leq M$, the first assertion in Theorem 1 remains valid under the complete intersection hypothesis about the $s_{j}$ 's on $V$, which provides some kind of robustness for multiplicative residue calculus involving Coleff-Herrera currents (see for example Proposition 3 below). The proof can be carried in a similar way than to that in [28], taking advantage of the wedge product operation introduced in [12] (see also [32]). Be careful however that the definition of the sheaf $\mathrm{CH}_{\mathcal{X}, V}(\cdot, E)$, whose global sections are $(0, M) E$-valued currents in the ambient manifold $\mathcal{X}$ with support lying in $V$, depends on the embedding $V \subset \mathcal{X}$. Nevertheless, Remark 1 will be important with respect to the role of such Coleff-Herrera residue currents in the construction of analytic tools for division theory, in accordance with that played by integration currents in intersection theory (see subsection 2.2 below).

We considered so far the holomorphic sections $s_{1}, \ldots, s_{m}$ of the hermitian line bundles $\left(\mathcal{O}_{\mathcal{X}}\left(\Delta_{j}\right),||_{j}\right)$ independently, then introduced the ColeffHerrera current $\bigwedge_{j=1}^{m} \bar{\partial}\left(1 / s_{j}\right) \wedge[V]$ through a multiplicative procedure which is reminiscent of the multiplicative operational formalism carried by the Cauchy kernel $d z_{1} / z_{1} \wedge \cdots \wedge d z_{m} / z_{m}$. Instead of that, one could interpret $s=s_{1} \oplus \cdots \oplus s_{m}$ as an holomorphic section of the $m$-holomorphic bundle $\bigoplus_{j=1}^{m} \mathcal{O}_{\mathcal{X}}\left(\Delta_{j}\right)$, equipped with the metric \|\|$^{2}=||_{1}^{2} \oplus \cdots \oplus||_{m}^{2}$. The robustness of the approach towards the current $\bigwedge_{j=1}^{m} \bar{\partial}\left(1 / s_{j}\right) \wedge[V]$ (in the complete intersection setting, see Theorem 1) motivates this alternative one. We still use analytic continuation, but this time respect to a single auxiliary complex parameter $\lambda$ instead of several. Such an approach was introduced in $[9,27]$, then extensively developed (after being interpreted within the frame of holomorphic hermitian bundles) in $[3,4,6,7]$. It is based on an averaging procedure. 
Theorem 2 (Bochner-Martinelli approach). Let $\mathcal{X}, V$, the $\Delta_{j}$ 's and the $s_{j}$ 's be as in the preamble of this section. Let \|\| be an arbitrary hermitian metric on the m-dimensional bundle $\bigoplus_{j=1}^{m} \mathcal{O}_{\mathcal{X}}\left(\Delta_{j}\right)=E_{\Delta}, s=s_{1} \oplus \cdots \oplus s_{m}$, and $s^{*}$ be the conjugate section of $s$, that is $s^{*}(x)(\xi)=\langle\langle\xi, s(x)\rangle\rangle_{x}$ for $x \in \mathcal{X}$, $\xi \in E_{\Delta, x}$. The holomorphic $\bigoplus_{r=0}^{m}{ }^{\prime} \mathcal{D}^{M, M+r}\left(\mathcal{X}, \bigwedge^{r} E_{\Delta}^{*}\right)$-valued map

$$
\lambda \longmapsto\left(\left(1-\|s\|^{2 \lambda}\right)+\bar{\partial}\|s\|^{2 \lambda} \wedge\left(\sum_{r=1}^{m} \frac{1}{(2 i \pi)^{r}} \frac{s^{*} \wedge\left(\bar{\partial} s^{*}\right)^{r-1}}{\|s\|^{2 r}}\right)\right) \wedge[V]
$$

(defined for $\operatorname{Re} \lambda>>1$ ) extends as an holomorphic map in a half-plane $\operatorname{Re} \lambda>-\eta$ for some $\eta>0$. It coincides at $\lambda=0$ coincides with its ${ }^{\prime} \mathcal{D}^{M, M+m}\left(\mathcal{X}, \wedge^{m} E_{\Delta}^{*}\right)$ component, that is, independently of the choice of the metric \|\| on $E_{\Delta}$, the Coleff-Herrera current $\bigwedge_{j=1}^{m} \bar{\partial}\left(1 / s_{j}\right) \wedge[V]$.

Remark 2. In the particular case where all line bundles $\mathcal{O}_{\mathcal{X}}\left(\Delta_{j}\right), j=1, \ldots, m$, are trivial over $\mathcal{X}$, and the holomorphic sections $s_{j}$ are holomorphic functions in $\mathcal{X}$ defining a complete intersection on $V$, one can interpret the current

$$
\left[\left(\left(1-\|s\|^{2 \lambda}\right)+\bar{\partial}\|s\|^{2 \lambda} \wedge\left(\sum_{r=1}^{m} \frac{1}{(2 i \pi)^{r}} \frac{s^{*} \wedge\left(\bar{\partial} s^{*}\right)^{r-1}}{\|s\|^{2 r}}\right)\right) \wedge[V]\right]_{\lambda=0}
$$

(now \|\| is the euclidean norm on $\mathbb{C}^{m}, s^{*}(x)(\xi)=\sum_{1}^{m} \overline{s_{j}(x)} \xi_{j}$ for $\xi \in \mathbb{C}^{m}$ ) as an averaged value of the currents

$$
\left[\bigwedge_{j=m}^{1}\left(1-\left|\left\langle u^{j}, s\right\rangle\right|^{2 \lambda_{j}}+\frac{1}{2 i \pi} \bar{\partial}\left(\frac{\left|\left\langle u^{j}, s\right\rangle\right|^{2 \lambda_{j}}}{\left\langle u^{j}, s\right\rangle}\right)\right) \wedge[V]\right]_{\lambda_{1}=\cdots=\lambda_{m}=0}
$$

(where $\left(u^{1}, \ldots, u^{m}\right) \in\left(\mathbb{P}^{n-1}(\mathbb{C})\right)^{m}$, and $\left\langle s, u^{j}\right\rangle=\sum_{k=0}^{m} u_{k}^{j} s_{k}$ for $\left.k=1, \ldots, m\right)$ defined as in in $(1)$, for $\left(u^{1}, \ldots, u^{m}\right)$ generic in $\left(\mathbb{P}^{m-1}(\mathbb{C})\right)^{m}$, respect to the normalized tensorized Fubini-Study metric on $\left(\mathbb{P}^{n-1}(\mathbb{C})\right)^{m}$.

Averaging Coleff-Herrera currents (realized in a multiplicative form as in Theorem 1) prevents usually from keeping track of the algebraic or arithmetic structure of the data (when there is one). Keeping track of such a structure is indeed better possible through a multiplicative approach such as in Theorem 1. Nevertheless, within the complete intersection frame, the fact that the current $\bigwedge_{j=1}^{m} \bar{\partial}\left(1 / s_{j}\right) \wedge[V]$ remains preserved under such averaging (Theorem 2) allows to reinterpret in geometric terms (and prove) the key (algebraic) operational property of multivariate residue calculus (see for example $[23,10,18]$ ), the so called Transformation Law. In order to formulate such a computational rule (which appears to be the geometric counterpart of Wiebe's theorem [33]) in a rather general form, one needs to introduce two sequences of Cartier divisors on the ambient complex manifold $\mathcal{X}$, namely $\left(\Delta_{1}, \ldots, \Delta_{m}, \mathfrak{T}_{0}, \ldots, \mathfrak{T}_{k-1}\right)$ and $\left(\widetilde{\Delta}_{1}, \ldots, \widetilde{\Delta}_{m}\right)(m+k \leq n-M)$, together with respective holomorphic sections $\left(s_{1}, \ldots, s_{m}, t_{0}, \ldots, t_{k-1}\right)=(s, t),\left(\widetilde{s_{1}}, \ldots, \widetilde{s_{m}}\right)=\widetilde{s}$; when $k=0$, one 
takes $\left\{\mathfrak{T}_{0}, \ldots, \mathfrak{T}_{k-1}\right\}=\emptyset$. The geometric hypothesis are :

$$
\begin{aligned}
& \operatorname{codim}_{V}\left(V \cap s^{-1}(0)\right)=\operatorname{codim}_{V}\left(V \cap \widetilde{s}^{-1}(0)\right)=m ; \\
& \overline{\left(V \cap s^{-1}(0)\right) \cap t_{l}^{-1}(0)}=V \cap s^{-1}(0) \quad \forall l=0, \ldots, k-1 ; \\
& \operatorname{codim}_{V}\left(V \cap s^{-1}(0) \cap t^{-1}(0)\right)=\operatorname{codim}_{V}\left(V \cap \widetilde{s}^{-1}(0) \cap t^{-1}(0)\right)=m+k \text {. }
\end{aligned}
$$

Let $E_{\Delta}=\bigoplus_{j=1}^{m} \mathcal{O}_{\mathcal{X}}\left(\Delta_{j}\right)$ and $E_{\widetilde{\Delta}}=\bigoplus_{j=1}^{m} \mathcal{O}_{\mathcal{X}}\left(\widetilde{\Delta}_{j}\right)$. As a pendant algebraic hypothesis, one assumes that there is a meromorphic section $H$ of $\operatorname{Hom}_{\mathbb{C}}\left(E_{\Delta}, E_{\widetilde{\Delta}}\right)$, together with positive integers $\nu_{0}, \ldots, \nu_{k}$, such that $\widetilde{s}=H \cdot s$ and

$$
\mathfrak{D}=\operatorname{det} H \otimes \bigotimes_{\kappa=0}^{k-1} t_{\kappa}^{\otimes \nu_{\kappa}} \in \mathcal{O}_{\mathcal{X}}\left(\mathcal{X}, \operatorname{Hom}_{\mathbb{C}}\left(E_{\Delta}, E_{\widetilde{\Delta}}\right) \otimes \bigotimes_{\kappa=0}^{k-1} \mathcal{O}_{\mathcal{X}}\left(\nu_{\kappa} \mathfrak{T}_{\kappa}\right)\right) .
$$

Theorem 3 (Transformation Law for Coleff-Herrera residue currents). Under the above geometric and algebraic hypothesis (4) and (5), one has the following identity between bundle valued currents :

$$
\bigwedge_{j=1}^{m} \bar{\partial}\left(\frac{1}{s_{j}}\right) \wedge \bigwedge_{\kappa=0}^{k-1} \bar{\partial}\left(\frac{1}{t_{\kappa}}\right) \wedge[V]=\left(\bigwedge_{j=1}^{m} \bar{\partial}\left(\frac{1}{\widetilde{s}_{j}}\right) \wedge \bigwedge_{\kappa=0}^{k-1} \bar{\partial}\left(\frac{1}{t_{\kappa}^{\nu_{\kappa}+1}}\right) \wedge[V]\right) \otimes \mathfrak{D}
$$

Proof. In order to get this result, we introduce metrics ||$_{\kappa}$ on the divisors $\mathfrak{T}_{\kappa}, \kappa=0, \ldots, k-1$, and compare the values at $\mu_{0}=\cdots=\mu_{k-1}$ (following the analytic continuation) of the two holomorphic maps (for $\operatorname{Re} \mu_{\kappa}>>1$, $\kappa=0, \ldots, k-1)$ :

$$
\begin{aligned}
& \mu \longmapsto \bigwedge_{\kappa=k-1}^{0} \bar{\partial}\left(\frac{\left|t_{\kappa}\right|_{\kappa}^{2 \mu_{\kappa}}}{t_{\kappa}}\right) \wedge \bigwedge_{j=1}^{m} \bar{\partial}\left(\frac{1}{s_{j}}\right) \wedge[V] \\
& \mu \longmapsto\left(\bigwedge_{\kappa=k-1}^{0} \bar{\partial}\left(\frac{\left|t_{\kappa}\right|_{\kappa}^{2 \mu_{\kappa}}}{t_{\kappa}}\right) \wedge \bigwedge_{j=1}^{m} \bar{\partial}\left(\frac{1}{\widetilde{s}_{j}}\right) \wedge[V]\right) \otimes \frac{\mathfrak{D}}{t_{0}^{\nu_{0}} \cdots t_{k-1}^{\nu_{k-1}}}
\end{aligned}
$$

These two current valued maps coincide for $\operatorname{Re} \mu_{\kappa}>>1, \kappa=0, \ldots, k-1$, because of the robustness assumption in Theorem 2, together with the holonomy and the Standard Extension Property of sections of the Coleff-Herrera sheaves $\mathrm{CH}_{\mathcal{X}, V \cap s^{-1}(0)}(\cdot ; \star S)$ or $\mathrm{CH}_{\mathcal{X}, V \cap \widetilde{s}^{-1}(0)}(\cdot ; \star S)$, where $S=\bigcup_{\kappa=0}^{k-1} t_{\kappa}^{-1}(0)$ (see [32], condition 1 in Definition 2). The fact that they share the same value at $\mu=0$ about any $x \in V \cap s^{-1}(0) \cap t^{-1}(0)$ follows from the robustness of the approach towards the Coleff-Herrera residue via analytic continuation (Theorem 1). On the other hand, both maps (6) vanish at $\mu=0$ about any $x \in V \cap\left(\widetilde{s}^{-1}(0) \backslash s^{-1}(0)\right) \cap t^{-1}(0)$ because of Cramer's rule, combined with the local duality property (2) in Theorem 1 (here is the crucial algebraic point on which relies Wiebe's theorem in the algebraic context). 
In order to focus on the importance of the Transformation Law, let us conclude this section with few comments inspired by arithmetic considerations. Let $\left\{p_{1}, \ldots, p_{\mathfrak{m}}\right\}$ be a collection of polynomials in $\mathbb{Z}\left[X_{1}, \ldots, X_{\mathfrak{n}}\right]$ (here $\mathfrak{m} \leq \mathfrak{n}$ ), with respective degrees $d_{1}, \ldots, d_{\mathfrak{m}}$, whose homogenizations define a complete intersection in $\mathbb{C}^{\mathfrak{n}}=\mathbb{P}^{\mathfrak{n}} \backslash\left\{z_{0}=0\right\}$. Denote as $T\left[p_{1}, \ldots, p_{\mathfrak{m}}\right]$ the $\bigwedge_{1}^{\mathfrak{m}} \mathcal{O}_{\mathbb{P}^{n}(\mathbb{C})}\left(-d_{j}\right)$-valued Coleff-Herrera global current in $\mathbb{P}^{\mathfrak{n}}(\mathbb{C})$ obtained from the current $T\left[p_{1}\right]=\bar{\partial}\left(1 / p_{1}\right)$ through the inductive process

$$
T\left[p_{1}, \ldots, p_{l+1}\right]=\left[\frac{1}{2 i \pi} \bar{\partial}\left(\frac{\left|p_{l+1}\right|_{l+1}^{2 \lambda}}{p_{l}}\right) \wedge T\left[p_{1}, \ldots, p_{l}\right]\right]_{\lambda=0}, l=1, \ldots, \mathfrak{m}-1 .
$$

The metric ||$_{l}$ one takes here on $\mathcal{O}_{\mathbb{P}^{\mathfrak{n}}(\mathbb{C})}\left(d_{l+1}\right)$ is the Fubini-Study metric, but this is in fact irrelevant, since the result after taking $\lambda=0$ in (7) is unaffected by such a choice. For $u^{1}, \ldots, u^{\mathfrak{n}-\mathfrak{m}}$ generic in $\mathbb{Z}^{\mathfrak{m}+1} \backslash\{0\}$, the linear subspace

$$
\Pi_{u}:=\left\{\left[z_{0}: \cdots: z_{\mathfrak{n}}\right] \in \mathbb{P}^{\mathfrak{n}}(\mathbb{C}) ;\left\langle u^{j}, z\right\rangle=0, j=1, \ldots, \mathfrak{m}\right\}
$$

is such that $\operatorname{dim}_{\mathbb{C}^{\mathfrak{n}}}\left(\Pi_{u} \cap\left\{p_{1}=\cdots=p_{\mathfrak{m}}=0\right\}\right)=0$. Consider the $\bigwedge_{1}^{\mathfrak{m}} \mathcal{O}_{\mathbb{P}^{\mathfrak{n}}(\mathbb{C})}\left(-d_{j}\right)$-valued $(\mathfrak{n}-\mathfrak{m}, \mathfrak{n}) \bar{\partial}$-closed current $T\left[p_{1}, \ldots, p_{\mathfrak{m}}\right] \wedge\left[\Pi_{u}\right]$. For any global smooth $(\mathfrak{m}, 0)$ form $\mathfrak{b}$ in $\mathbb{P}^{\mathfrak{n}}(\mathbb{C})$ with values in $\bigwedge_{1}^{\mathfrak{m}} \mathcal{O}_{\mathbb{P}^{\mathfrak{n}}}(\mathbb{C})\left(d_{j}\right)$, one has,

$$
\begin{aligned}
& \left\langle T\left[p_{1}, \ldots, p_{\mathfrak{m}}\right] \wedge\left[\Pi_{u}\right], \mathfrak{b}\right\rangle \\
& =\left\langle\left[\left(1-\left|z_{0}\right|^{2 \lambda}\right) T\left[p_{1}, \ldots, p_{\mathfrak{m}}\right] \wedge\left[\Pi_{u}\right]\right]_{\lambda=0}, \mathfrak{b}\right\rangle \\
& \quad+\left\langle\left[\left|z_{0}\right|^{2 \lambda} T\left[p_{1}, \ldots, p_{\mathfrak{m}}\right] \wedge\left[\Pi_{u}\right]\right]_{\lambda=0}, \mathfrak{b}\right\rangle \\
& =\left\langle\left(T\left[p_{1}, \ldots, p_{\mathfrak{m}}\right] \wedge\left[\Pi_{u}\right]\right)_{\mid\left\{z_{0}=0\right\}}, \mathfrak{b}\right\rangle+\left\langle\left(T\left[p_{1}, \ldots, p_{\mathfrak{m}}\right] \wedge\left[\Pi_{u}\right]\right)_{\mid \mathbb{C}_{\mathfrak{n}}}, \mathfrak{b}\right\rangle .
\end{aligned}
$$

An explicit computation of

$$
\left\langle\left(T\left[p_{1}, \ldots, p_{\mathfrak{m}}\right] \wedge\left[\Pi_{u}\right]\right)_{\mid \mathbb{C}^{\mathfrak{n}}}, \mathfrak{b}\right\rangle
$$

can be carried thanks to the Transformation Law (such as formulated in Theorem 3 with $k=0$ ), following the procedure which is described in [18]. In particular, if $\mathfrak{b}[q, J]$ is the form expressed in $\mathbb{C}^{\mathfrak{n}}$, in affine coordinates $\zeta_{1}, \ldots, \zeta_{\mathfrak{n}}$, as

$$
\mathfrak{b}[q, J]=\frac{q(\zeta)}{\left(1+|\zeta|^{2}\right)^{(d+\operatorname{deg} q) / 2}} \bigwedge_{l=1}^{\mathfrak{m}} d\left(\frac{\zeta_{j_{l}}}{\sqrt{1+|\zeta|^{2}}}\right), d=d_{1}+\cdots+d_{\mathfrak{m}}
$$

for some $q \in \mathbb{Z}\left[X_{1}, \ldots, X_{\mathfrak{n}}\right]$ and $1 \leq j_{1}<\cdots<j_{\mathfrak{m}} \leq \mathfrak{n}$, one gets for (8) a somehow explicit rational expression $R_{q, J}[u] \in \mathbb{Q}$. A key point in such a procedure is that it provides a final estimate on the logarithmic height $h$ of the rational function $R_{q, J}[u]$ of the form 


$$
h\left(R_{q, J}[u]\right) \leq h(q)+\kappa_{1}(n) D(D+\operatorname{deg} q) \max \left(h\left(p_{j}\right), \log \left\|u^{j}\right\|\right),
$$

where $D=\prod_{1}^{\mathfrak{m}}\left(d_{j}+1\right)$, in accordance with the geometric Bézout theorem and its arithmetic counterpart. If $q\left(T\left[p_{1}, \ldots, p_{\mathfrak{m}}\right] \wedge\left[\Pi_{u}\right]\right)_{\left\{z_{0}=0\right\}}$, one gets a rational expression with logarithmic height control (9) for $\left\langle T\left[p_{1}, \ldots, p_{\mathfrak{m}}\right] \wedge\left[\Pi_{u}\right], \mathfrak{b}[q, J]\right\rangle$.

\section{Coleff-Herrera residue currents and ordered sequences of sections of Cartier divisors}

Let $\mathcal{X}$ be an $n$-dimensional complex manifold. Given a purely dimensional cycle $[V] \in Z^{n-M}(\mathcal{X})$ (identified here with the $(M, M)$ associated integration current on it and with support denoted as $V$ ) and a closed submanifold $\mathcal{Z} \subset \mathcal{X}$, the splitting operation

$$
[V]=[V]^{\mathcal{Z}}+[V]^{\mathcal{X} \backslash \mathcal{Z}}
$$

which consists in separating (as components of $[V]^{\mathcal{Z}}$ ) the components of $[V]$ with support lying in $\mathcal{Z}$, from the others, is one of the major operational tools in geometric intersection theory. Transposed in algebraic terms, it leads to the notion of gap sheaf (see for an introduction to the subject Part I in $[26])$.

Example 1. $[30,31,24]$ Let $\left[V_{1}\right], \ldots,\left[V_{\mathfrak{m}}\right]$ be $\mathfrak{m}$ purely dimensional algebraic cycles in $\mathbb{P}^{\mathfrak{n}}(\mathbb{C}),[V]$ be the $(n-M)$-cycle in $\mathcal{X}=\mathbb{P}^{n}(\mathbb{C})(n=\mathfrak{m}(\mathfrak{n}+1)-1$, $\left.M=\sum_{j=1}^{\mathfrak{m}} \operatorname{codim}_{\mathbb{P n}(\mathbb{C})} V_{j}\right)$ corresponding to the ruled join

$$
J\left(V_{1}, \ldots, V_{\mathfrak{m}}\right)=\left\{\left[Z_{1}: \cdots: Z_{\mathfrak{m}}\right] \in \mathbb{P}^{\mathfrak{m}(\mathfrak{n}+1)-1}(\mathbb{C}) ; z_{j} \in V_{j} \quad \forall j=1, \ldots, \mathfrak{m}\right\}
$$

(multiplicities been taken into account), and $\mathcal{Z}$ be the diagonal subspace of $\mathbb{P}^{n}(\mathbb{C})$ defined as a complete intersection as the set of points $\left[Z_{1}: \cdots: Z_{\mathfrak{m}}\right] \in$ $\mathbb{P}^{n}(\mathbb{C})$ such that $Z^{k}=Z^{1}$ for any $k \in\{2, \ldots, \mathfrak{m}\}$ (see [19]). Note that, in this

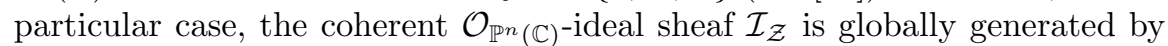
$(\mathfrak{m}-1)(\mathfrak{n}+1)$ holomorphic sections $\sigma_{l}$ of the same line bundle $\mathcal{O}_{\mathbb{P}^{n}(\mathbb{C})}(1)$. Moreover the zero sets $\sigma_{l}^{-1}(0), l=1, \ldots,(\mathfrak{m}-1)(\mathfrak{n}+1)$, define here $\mathcal{Z}$ as a complete intersection in the ambient $n$-dimensional manifold $\mathcal{X}=\mathbb{P}^{n}(\mathbb{C})$.

What we propose to do now is to transpose within the frame of global sections of the Coleff-Herrera sheaves $\mathrm{CH}_{\mathcal{X}, V}$ (or $\mathrm{CH}_{\mathcal{X}, V}(\cdot ; \star S)$ ) (instead of the geometric frame of integration currents) the splitting operation (10), together with the construction such an operation generates when applied inductively. We should point out here that such a construction is directly inspired from the ideas introduced in [13] by N. Coleff and M. Herrera (see for example [29]). 


\subsection{The Coleff-Herrera product}

Let $\mathcal{X}$ and $V$ be as in Section 1 . Consider also an ordered sequence $\Delta_{1}, \ldots, \Delta_{m}$ (one drops here the assumption $m \leq n-M$ ) of Cartier divisors on $\mathcal{X}$, equipped respectively with hermitian metrics ||$_{j}, j=1, \ldots, m$, and with holomorphic global sections $s_{1}, \ldots, s_{m}$. The major difference with Section 1 is that we forget here the geometric assumption that the closed hypersurfaces $s_{j}^{-1}(0) \subset \mathcal{X}, j=1, \ldots, m$, intersect as a complete intersection on the closed analytic subset $V$. Let $T$ be a global section over $\mathcal{X}$ of the Coleff-Herrera sheaf $\mathrm{CH}_{\mathcal{X}, V}(\cdot, E)$, where $E$ denotes some finite rank holomorphic bundle over $\mathcal{X}$. Let $\bigwedge_{j=1}^{m} \bar{\partial}\left(1 / s_{j}\right) \wedge T$ be the current in

$$
\bigoplus_{r=0}^{m} \bigoplus_{1 \leq j_{1}<\cdots<j_{r} \leq m}{ }^{\prime} \mathcal{D}^{(0, M+r)}\left(\mathcal{X}, \bigwedge_{l=1}^{r} \mathcal{O}_{\mathcal{X}}\left(-\Delta_{j_{l}}\right) \otimes E\right)
$$

defined inductively from

$$
\bar{\partial}\left(\frac{1}{s_{1}}\right) \wedge T=\left[\left(1-\left|s_{1}\right|_{1}^{2 \lambda}+\frac{1}{2 i \pi} \bar{\partial}\left(\frac{\left|s_{1}\right|_{1}^{2 \lambda}}{s_{1}}\right)\right) \wedge T\right]_{\lambda=0}
$$

by the iterative process

$$
\bigwedge_{j=1}^{l+1} \bar{\partial}\left(\frac{1}{s_{j}}\right) \wedge T=\left[\left(1-\left|s_{l+1}\right|_{l+1}^{2 \lambda}+\frac{1}{2 i \pi} \bar{\partial}\left(\frac{\left|s_{l+1}\right|_{l+1}^{2 \lambda}}{s_{l+1}}\right)\right) \wedge \bigwedge_{j=1}^{l} \bar{\partial}\left(\frac{1}{s_{j}}\right) \wedge T\right]_{\lambda=0}
$$

for $l=1, \ldots, m-1$. Remark that in spite of the notation $\bigwedge_{j=1}^{m} \bar{\partial}\left(1 / s_{j}\right) \wedge T$ (that one uses here for the sake of simplicity), this current is not in general a $(0, m+M)$ current. The above inductive construction is independent of the choice of the metrics ||$_{j}$ on the line bundles $\mathcal{O}_{\mathcal{X}}\left(\Delta_{j}\right)$, but of course depends on the ordering of the sequence of divisors $\left\{\Delta_{1}, \ldots, \Delta_{m}\right\}$. When the hypersurfaces $s_{j}^{-1}(0), j=1, \ldots, m$, define a complete intersection on $V$, this current coincides with its ${ }^{\prime} \mathcal{D}^{(0, M+m)}\left(\mathcal{X}, \bigwedge_{j=1}^{m} \mathcal{O}_{\mathcal{X}}\left(-\Delta_{j}\right) \otimes E\right)$ component, and can be recovered in a robust way in a neighborhood of $V \cap s^{-1}(0)$ as the value at $\lambda_{1}=\cdots=\lambda_{m}=0$ of an holomorphic function in $m$ variables in a product of half planes $\operatorname{Re} \lambda_{j}>-\eta$ for some $\eta>0$ (see Remark 1 above). The iterative construction is justified by the local structure theorems for sections of the Coleff-Herrera sheaves $\mathrm{CH}_{\mathcal{X}, W}(\cdot ; \star S, E)$, where $W$ denotes a purely dimensional closed analytic subset of $V$ and $S$ a closed hypersurface in a neighborhood of $W$ in $\mathcal{X}$ such that $\overline{W \backslash S}=W$. When $\underline{j}=\left(j_{1}, \ldots, j_{r}\right)$ is a $r$-uplet $(0 \leq r \leq m, \underline{j}=\emptyset$ if $r=0)$ of strictly increasing integers $1 \leq j_{1}<\cdots<j_{r} \leq m$, the component $\left(\bigwedge_{j=1}^{m} \bar{\partial}\left(1 / s_{j}\right) \wedge T\right)_{j}$ in ${ }^{\prime} \mathcal{D}^{(0, M+r)}\left(\mathcal{X}, \bigwedge_{l=1}^{r} \mathcal{O}_{\mathcal{X}}\left(-\Delta_{j_{l}}\right) \otimes E\right)$ is a global section of the Coleff-Herrera sheaf $\mathrm{CH}_{\mathcal{X}, V_{\underline{j}}[s]}\left(\cdot ; \star S_{\underline{j}}[s], E_{\underline{j}}\right)$, where $E_{\underline{j}}=E_{\underline{j}}[\Delta, E]=\bigwedge_{l=1}^{r} \mathcal{O}_{\mathcal{X}}\left(-\Delta_{j_{l}}\right) \otimes E$, $V_{\underline{j}}[s]$ is a purely $(n-M-\bar{r})$-dimensional closed subset of $V$ and $S_{\underline{j}}[s]$ some 
closed hypersurface in a neighborhood of $V_{\underline{j}}[s]$ such that $\overline{V_{\underline{j}}[s] \backslash S_{\underline{j}}[s]}=V_{\underline{j}}[s]$. The construction of $V_{j}[s]$ is carried through the procedure that leads to the construction of the multi-cycle of contact (see [31]) between a given cycle $[V]$ and a smooth closed submanifold $Z \subset \mathcal{X}$ contained in the intersection of an ordered sequence $H_{1}, \ldots, H_{m}(m \leq \operatorname{dim} V)$ of closed hypersurfaces. More precisely, $V_{j}[s]$ can be reached through the following iterated splitting operation : when $W$ is a closed purely dimensional subset in $\mathcal{X}$ and $S$ denotes a closed hypersurface in a neighborhood of $W$, then $W$ is (geometrically) decomposed as

$$
W=W^{S} \cup W^{\mathcal{X} \backslash S},
$$

where $W^{S}$ denotes the union of irreducible components of $W$ lying entirely in $S$ and $W^{\mathcal{X} \backslash S}$ the union of the remaining ones. The closed analytic set $V_{j}[s]$ appears then as the end term in the inductive sequence :

$$
\begin{aligned}
& V_{\underline{j}, 1}[s]=V^{s_{1}^{-1}(0)} \text { if } 1 \notin\left\{j_{1}, \ldots, j_{r}\right\} \text {; else } V_{\underline{j}, 1}[s]=V^{\mathcal{X} \backslash s_{1}^{-1}(0)} \cap s_{1}^{-1}(0) ; \\
& \begin{array}{llll}
\text {. } & \ldots & \ldots
\end{array} \\
& V_{\underline{j}, k+1}[s]=\left(V_{\underline{j}, k}[s]\right)^{s_{k+1}^{-1}(0)} \text { if } k+1 \notin\left\{j_{1}, \ldots, j_{r}\right\} ; \\
& \text { else } V_{\underline{j}, k+1}[s]=\left(V_{\underline{j}, k}[s]\right)^{\mathcal{X} \backslash s_{k+1}^{-1}(0)} \cap s_{k+1}^{-1}(0) ; \\
& \begin{array}{lll}
\text {. } & \ldots & \ldots
\end{array} \\
& V_{\underline{j}}[s]=V_{\underline{j}, m}[s]=\left(V_{\underline{j}, m-1}[s]\right)^{s_{m}^{-1}(0)} \text { if } m \notin\left\{j_{1}, \ldots, j_{r}\right\} ; \\
& \text { else } V_{\underline{j}}[s]=V_{\underline{j}, m}[s]=\left(V_{\underline{j}, m-1}[s]\right)^{\mathcal{X} \backslash s_{m}^{-1}(0)} \cap s_{m}^{-1}(0) .
\end{aligned}
$$

If $m \leq n-M$ and $r=m, V_{\{1, \ldots, m\}}[s]$ is the so-called essential intersection $\left(V \cap s_{1}^{-1}(0) \cap \cdots \cap s_{m}^{-1}(0)\right)_{\text {ess }}$ while, if $r=0, V_{\emptyset}[s]$ is the union of the irreducible components of $V$ which lie entirely in the intersection $s_{1}^{-1}(0) \cap \cdots \cap s_{m}^{-1}(0)$.

The same iterative procedure allows also to define the Coleff-Herrera current $\bigwedge_{j=1}^{m} \bar{\partial}\left(1 / s_{j}\right) \wedge \mathcal{T}$, when $\mathcal{T}$ is a global section of the Coleff-Herrera sheaf $\mathrm{CH}_{\mathcal{X}, V}(\cdot ; \star S, E)$ for some closed hypersurface $S$ in a neighborhood of $V$ such that $\overline{V \backslash S}=V$. As before, each component $\left(\bigwedge_{j=1}^{m} \bar{\partial}\left(1 / s_{j}\right) \wedge \mathcal{T}\right)_{j}$ is then a global section of the Coleff-Herrera sheaf $\mathrm{CH}_{\mathcal{X}, V_{j}[s]}\left(\cdot ; \star S_{j}[s, S], E_{\underline{j}}\right)$ for some convenient hypersurface $S_{\underline{j}}[s, S]$ in a neighborhood of $V_{\underline{j}}[s]$ such that $\overline{V_{\underline{j}}[s] \backslash S_{\underline{j}}[s, S]}=V_{\underline{j}}[s]$. One can point out (see [8]) that analytic continuation with respect to a single complex parameter (as in Theorem 2) provides a direct approach to such a current.

Proposition 1. Let $\mathcal{X}, V$, the $\Delta_{j}$ 's, the metrics ||$_{j}$, the holomorphic sections $s_{j}$ 's, and the Coleff-Herrera current $\mathcal{T}$ (with eventual poles) be as above. Let $\gamma_{1}>\gamma_{2}>\cdots>\gamma_{m} \geq 1$ be $m$ positive integers and $\epsilon>0$. Then the currentvalued map 


$$
\begin{array}{r}
\lambda \in\left\{\lambda \in \mathbb{C} ;\left|\arg _{] \pi, \pi[}(\lambda)\right|<\frac{\pi}{2\left(\gamma_{1}+\epsilon\right)} ;|\lambda|>>1\right\} \\
\longmapsto\left[\bigwedge_{j=m}^{1}\left(1-\left|s_{j}\right|_{j}^{2 \lambda^{\gamma_{j}}}+\frac{1}{2 i \pi} \bar{\partial}\left(\frac{\left|s_{j}\right|_{j}^{2 \lambda^{\gamma_{j}}}}{s_{j}}\right)\right)\right] \wedge \mathcal{T}
\end{array}
$$

extends as a holomorphic map in an open neighborhood of the closed sector $\left\{\left|\arg _{] \pi, \pi[}(\lambda)\right| \leq \pi /\left(2 \gamma_{1}\right)\right\}$. Its value at $\lambda=0$ equals the current $\bigwedge_{j=1}^{m} \bar{\partial}\left(1 / s_{j}\right) \wedge$ $\mathcal{T}$.

We intend from now on to focus on the advantages (or disadvantages) that carries the idea of averaging (as done in Section 1 when stating Theorem 1, then Theorem 2) Coleff-Herrera residues of the form $\bigwedge_{j=1}^{m} \bar{\partial}\left(1 / s_{j}\right) \wedge T$ or $\bigwedge_{j=1}^{m} \bar{\partial}\left(1 / s_{j}\right) \wedge \mathcal{T}$, when $V$ is a purely dimension $(n-M)$-closed analytic subset in a complex manifold $\mathcal{X}, s_{1}, \ldots, s_{m}$, being holomorphic sections of arbitrary Cartier divisors on $\mathcal{X}$.

\subsection{Vogel sequences and Vogel residue currents}

The first positive point with respect to the averaging idea arises from the theory of improper intersection on $\mathcal{X}$, as developed in the algebraic context in [30] (see also [24]), and in the analytic context in [31]. Let $\mathcal{X}$ and $V$ be as in Section 1, and $\mathcal{I}$ be a coherent ideal sheaf in $\mathcal{O}_{\mathcal{X}}$, with $Z(\mathcal{I})$ being the support of the quotient sheaf $\mathcal{O}_{\mathcal{X}} / \mathcal{I}$. We recall that, at the local level, a local Vogel sequence (in the geometric sense) at $x \in V$ (for $\mathcal{I}_{x}$, on the germ of complex analytic space $\left(V_{x},\left(\mathcal{O}_{V}\right)_{x}\right)$ is a sequence $\left(s_{1, x}, \ldots, s_{n-M, x}\right)$ in the ideal $\mathcal{I}_{x}$ such that there is a neighborhood $U_{x}$ of $x$ in $\mathcal{X}$, together with representatives $s_{1, x}, \ldots, s_{n-M, x}$ of the germs in this neighborhood, with

$$
\operatorname{codim}_{V}\left[\left(\left(U_{x} \cap V\right) \backslash Z(\mathcal{I})\right) \cap \bigcap_{j=1}^{l} s_{j, x}^{-1}(0)\right]=l \text { or }+\infty, l=1, \ldots, n-M .
$$

Such geometric conditions (15) (considered in the semi global setting) are already sufficient in order to imply the following Proposition.

Proposition 2. Let $U$ be an open set in $\mathbb{C}^{n}, V$ be a purely $M$-codimensional closed analytic subset of $U, I$ be an ideal in $\mathcal{O}_{\mathbb{C}^{n}}(U)$ (with zero set $Z(I)$ in $U$ and generators $\left.\sigma_{0}, \ldots, \sigma_{L}\right)$. Let $\left(s_{1}, \ldots, s_{n}\right)$ be a sequence of elements in $I$ such that

$$
\operatorname{codim}_{V}\left[(V \backslash Z(I)) \cap \bigcap_{j=1}^{l} s_{j}^{-1}(0)\right]=l \text { or }+\infty, l=1, \ldots, n-M .
$$

If $T$ is an element in $\mathrm{CH}_{U, V}(U, \mathbb{C})$, then 


$$
\bigwedge_{j=1}^{n-M} \bar{\partial}\left(\frac{1}{s_{j}}\right) \wedge T=T_{\mid Z(I)}+\sum_{l=1}^{n-M} T\left[s_{1}, \ldots, s_{l}\right]_{\mid Z(I)},
$$

where $T\left[s_{1}, \ldots, s_{l}\right]$ is defined inductively after $l-1$ operations via

$$
\begin{aligned}
& T\left[s_{1}\right]=\frac{1}{(2 i \pi)}\left[\bar{\partial}\left(\frac{\left|s_{1}\right|^{2 \lambda}}{s_{1}}\right) \wedge T_{\mid U \backslash s_{1}^{-1}(0)}\right]_{\lambda=0} \\
& T\left[s_{1}, \ldots, s_{l^{\prime}+1}\right]=\frac{1}{(2 i \pi)}\left[\bar{\partial}\left(\frac{\left|s_{l^{\prime}}\right|^{2 \lambda}}{s_{l^{\prime}}}\right) \wedge T\left[s_{1}, \ldots, s_{l^{\prime}}\right]_{\mid U \backslash s_{l^{\prime}}^{-1}(0)}\right]_{\lambda=0} .
\end{aligned}
$$

Moreover all currents $T\left[s_{1}, \ldots, s_{l}\right], l=1, \ldots, n-M$, are $\bar{\partial}$-closed, and thus are Coleff-Herrera currents. Here $T_{\mid Z(I)}$ means $\left[\left(1-|\sigma|^{2 \lambda}\right) T\right]_{\lambda=0}$, while $T_{\mid U \backslash Z(I)}$ means $\left[|\sigma|^{2 \lambda} T\right]_{\lambda=0}$, when $T$ is a Coleff-Herrera current.

Remark 3. Formula (17) remains valid if $T$ is replaced by a meromorphic Coleff-Herrera current $\mathcal{T} \in \mathrm{CH}_{U, V}(U ; \star S, \mathbb{C})$ for some closed hypersurface $S$ in $U$ such that $\overline{V \backslash S}=V$. But the second assertion does not remain true.

Remark 4. All currents $\left(T\left[s_{1}, \ldots, s_{l}\right]\right)_{\mid Z(I)}$ are supported, as the current $T_{\mid Z(I)}$, by the closed analytic set $V \cap Z(I)$. As noticed in [7], such a $(0, l+M)$ current $\left(T\left[s_{1}, \ldots, s_{l}\right]\right)_{\mid Z(I)}$ vanishes as soon as $l+M<\operatorname{codim}(V \cap Z(I))$, which means that only the terms with index $l$ between $\operatorname{codim}(V \cap Z(I))-M$ and $n-M$ remain in the development (17). The first term $T_{\mid Z(I)}$ is of course only present if $\operatorname{codim}(V \cap Z(I))=\operatorname{codim} V=M$.

Proof. Decompose $T$ as

$$
T=\left[\left(1-\left|s_{1}\right|^{2 \lambda}\right) T\right]_{\lambda=0}+\left[\left|s_{1}\right|^{2 \lambda} T\right]_{\lambda=0}=T_{\mid s_{1}^{-1}(0)}+T_{\mid U \backslash s_{1}^{-1}(0)} .
$$

The fact that $T_{\mid s_{1}^{-1}(0)}$ has its support included in $V \cap s_{1}^{-1}(0)$ implies

$$
\bar{\partial}\left(\frac{1}{s_{1}}\right) \wedge T_{\mid s_{1}^{-1}(0)}=\left[\left(1-\left|s_{1}\right|^{2 \lambda}+\frac{1}{2 i \pi} \bar{\partial}\left(\frac{\left|s_{1}\right|^{2 \lambda}}{s_{1}}\right)\right) \wedge T_{s_{1}^{-1}(0)}\right]_{\lambda=0}=T_{\mid s_{1}^{-1}(0)} .
$$

Since $T_{U \backslash s_{1}^{-1}(0)}$ is a Coleff-Herrera current with respect to $V^{U \backslash s_{1}^{-1}(0)}$, one has

$$
\bar{\partial}\left(\frac{1}{s_{1}}\right) \wedge T_{\mid U \backslash s_{1}^{-1}(0)}=\frac{1}{2 i \pi}\left[\bar{\partial}\left(\frac{\left|s_{1}\right|^{2 \lambda}}{s_{1}}\right) \wedge T_{U \backslash s_{1}^{-1}(0)}\right]_{\lambda=0}=T\left[s_{1}\right] .
$$

We now observe that, since all components in the support $V^{s_{1}^{-1}(0)}$ of $T_{\mid s_{1}^{-1}(0)}$ lie in $V \cap s_{1}^{-1}(0)$ and have codimension $M$ as $V$, the geometric condition (16) for $l=2$ implies 


$$
\bigwedge_{j=2}^{n-M} \bar{\partial}\left(\frac{1}{s_{j}}\right) \wedge T_{\mid s_{1}^{-1}(0)}=T_{\mid s_{1}^{-1}(0)}=T_{\mid Z(I)} .
$$

It remains to continue the process to compute

$$
\left[\left(1-\left|s_{2}\right|^{2 \lambda}+\frac{1}{2 i \pi} \bar{\partial}\left(\frac{\left|s_{2}\right|^{2 \lambda}}{s_{2}}\right)\right) \wedge T\left[s_{1}\right]\right]_{\lambda=0} .
$$

In order to do this, one decomposes $T\left[s_{1}\right]$ as in (18):

$T\left[s_{1}\right]=\left[\left(1-\left|s_{2}\right|^{2 \lambda}\right) T\left[s_{1}\right]\right]_{\lambda=0}+\left[\left|s_{2}\right|^{2 \lambda} T\left[s_{2}\right]\right]_{\lambda=0}=T\left[s_{1}\right]_{\mid s_{2}^{-1}(0)}+T\left[s_{1}\right]_{\mid U \backslash s_{2}^{-1}(0)}$.

The geometric condition (16) for $l=3$ implies now that

$$
\left[\left(1-\left|s_{2}\right|^{2 \lambda}+\frac{1}{2 i \pi} \bar{\partial}\left(\frac{\left|s_{2}\right|^{2 \lambda}}{s_{2}}\right)\right) \wedge T\left[s_{1}\right]_{\mid s_{2}^{-1}(0)}\right]_{\lambda=0}=T\left[s_{1}\right]_{\mid Z(I)} .
$$

The contribution

$$
\left[\left(1-\left|s_{2}\right|^{2 \lambda}+\frac{1}{2 i \pi} \bar{\partial}\left(\frac{\left|s_{2}\right|^{2 \lambda}}{s_{2}}\right)\right) \wedge T\left[s_{1}\right]_{\mid U \backslash s_{2}^{-1}(0)}\right]_{\lambda=0},
$$

equals, as for the first step,

$$
\left.\frac{1}{(2 i \pi}\left[\bar{\partial}\left(\frac{\left|s_{2}\right|^{2 \lambda}}{s_{2}}\right)\right) \wedge T\left[s_{1}\right]_{\mid U \backslash s_{2}^{-1}(0)}\right]_{\lambda=0}=T\left[s_{1}, s_{2}\right] .
$$

The procedure can be thus repeated, which leads to (17). In order to prove that all $T\left[s_{1}, \ldots, s_{l}\right]$ are $\bar{\partial}$ closed, it is enough to prove it for $T\left[s_{1}\right]$ (then the proof goes inductively). The result for $T\left[s_{1}\right]$ follows immediately from the fact that

$$
T\left[s_{1}\right]=\frac{1}{(2 i \pi)}\left[\bar{\partial}\left(\frac{\left|s_{1}\right|^{2 \mu}}{s_{1}}\right) \wedge\left[\left|s_{1}\right|^{2 \lambda} T\right]_{\lambda=0}\right]_{\mu=0},
$$

by computing $\bar{\partial}$ of both sides in (19).

Proposition 3. Let $U$ be an open set in $\mathbb{C}^{n}, M \leq n, f_{1}, \ldots, f_{M}$ be $M$ holomorphic functions in $U$, and $T=T[f]$ the $(0, M)$-Coleff-Herrera current

$$
T[f]=\left(\bigwedge_{j=1}^{M} \bar{\partial}\left(\frac{1}{f_{j}}\right)\right)_{(0, M)}
$$

(with respect to $\left.(V[f])_{\text {ess }}=\left(f_{1}^{-1}(0) \cap \cdots \cap f_{M}^{-1}(0)\right)_{\text {ess }}\right)$. Let $I$ be an ideal in $\mathcal{O}_{\mathcal{X}}(U)$, such that $\overline{(V[f])_{\text {ess }} \backslash Z(I)}=(V[f])_{\text {ess }}$, with generators $\left(\sigma_{0}, \ldots, \sigma_{L}\right)$. If $u^{1}, \ldots, u^{n-M}$, one after each other, in this order, are generic in $\mathbb{P}^{L}(\mathbb{C})$, such that in particular $\left(s_{1}, \ldots, s_{n-M}\right):=\left(\left\langle u^{1}, \sigma\right\rangle, \ldots,\left\langle u^{n-M}, \sigma\right\rangle\right)$ fulfills conditions 
(16) with $V=(V[f])_{\mathrm{ess}}$, then the current

$$
\bigwedge_{j=1}^{n-M} \bar{\partial}\left(\frac{1}{\left\langle u^{j}, \sigma\right\rangle}\right) \wedge T[f]
$$

is annihilated as a current by $I^{n-M}$.

Proof. Before presenting a sketch of the proof, let us focus on a simple situation (to which the general case will in fact be reduced). Suppose for the moment that $T \in \mathrm{CH}_{U, V}(U, \mathbb{C})$, and that $\sigma$ and $h$ are two holomorphic functions in a neighborhood of $V$, such that $(\sigma, h)$ defines a complete intersection on $V$. Then, we claim that the current

$$
\left[\left[\bar{\partial}\left(\frac{|\sigma h|^{2 \lambda}}{\sigma h}\right) \wedge T\right]_{\lambda=0}\right]_{\mid \sigma^{-1}(0)}=\left[\left(1-|\sigma|^{2 \mu}\right)\left[\bar{\partial}\left(\frac{|\sigma h|^{2 \lambda}}{\sigma h}\right) \wedge T\right]_{\lambda=0}\right]_{\mu=0}
$$

is annihilated by $\sigma$. This follows from the fact that the map

$$
\left(\lambda_{1}, \lambda_{2}\right) \in\left\{\operatorname{Re} \lambda_{1}>>1, \operatorname{Re} \lambda_{2}>>1\right\} \longmapsto \frac{|h|^{2 \lambda_{1}}}{h}|\sigma|^{\lambda_{2}} T
$$

extends as an holomorphic function of two variables to a product of half spaces $\left\{\operatorname{Re} \lambda_{1}>-\eta\right\} \times\left\{\operatorname{Re} \lambda_{2}>-\eta\right\}$ for some $\eta>0$. Since $\overline{V \backslash \sigma^{-1}(0)}=$ $\overline{V \backslash h^{-1}(0)}=V$, it follows that

$$
\left[\bar{\partial}\left(\frac{|h|^{2 \lambda_{1}}}{h}|\sigma|^{2 \lambda_{2}} T\right)\right]_{\lambda_{1}=\lambda_{2}=0}=\left[\bar{\partial}\left(\frac{|h|^{2 \lambda}}{h}\right) \wedge T\right]_{\lambda=0}=\bar{\partial}\left(\frac{T}{h}\right),
$$

which is a Coleff-Herrera current on $U$ with respect to $V \cap\{h=0\}$. Since $\sigma$ does not vanish identically on any component the set $V \cap\{h=0\}$, one has

$$
\left[\left(1-|\sigma|^{2 \mu}\right) \bar{\partial}\left(\frac{T}{h}\right)\right]_{\mu=0}=0,
$$

which proves the claim.

Consider now the Coleff Herrera current $T[f]$. Let $\widetilde{U} \stackrel{\pi}{\rightarrow} U$ be the normalized blowup of $U$ along $I$ and $\mathcal{E}(I)$ be its exceptional divisor (multiplicities being taken into account). Though $\widetilde{U}$ is not smooth, one can (locally) consider an embedding $\widetilde{U} \subset \Omega \subset \mathbb{C}^{N}$ and treat the current $\left(\bigwedge_{1}^{M} \bar{\partial}\left(1 / \pi^{*}\left[f_{j}\right]\right)\right)_{(0, M)}$ as the $\bar{\partial}$-closed $(N-n, M+N-n)$ current $\left(\bigwedge_{1}^{M} \bar{\partial}\left(1 / \pi^{*}\left[f_{j}\right]\right)\right)_{(0, M)} \wedge[\widetilde{U}]$ in $\Omega$. It factorizes in $\Omega$ as the product of a $(0, M+N-n)$ Coleff-Herrera current with eventual poles (with respect to some closed $M$-codimensional analytic subset $\widetilde{V}[f]$ of $\widetilde{U})$ times an holomorphic $(N-n, 0)$ form. Moreover, $\overline{\widetilde{V}[f] \backslash \pi^{-1}(Z(I))}=\widetilde{V}[f]$. Actually, one can approach the current $\left(\bigwedge_{1}^{M} \bar{\partial}\left(1 / \pi^{*}\left[f_{j}\right]\right)\right)_{(0, M)}$ by $\chi_{\left\|\pi^{*}(\sigma)\right\| \geq \epsilon} \bigwedge_{1}^{M} \bar{\partial}\left(1 / \pi^{*}\left[f_{j}\right]\right)$ when $\epsilon>0$ tends to 
0 , and $\chi$ denotes a smooth cutoff function as in [12]. The exceptional divisor $\mathcal{E}(I)$ defines then a divisor $\mathcal{E}(I)_{\mid \widetilde{V}[f]}$ on $\widetilde{V}[f]$. Choose $u^{1}$ (generically) such that $\pi^{*}\left[s^{1}\right]_{\mid \tilde{V}[f]}$ vanishes on each irreducible component of the support $\mathcal{E}(I)_{\mid \tilde{V}[f]}$ exactly at the multiplicity on this component of the divisor $\mathcal{E}(I)_{\mid \tilde{V}[f]}$. This means that $\pi^{*}\left[s_{1}\right]_{\mid \tilde{V}[f]}$ can be expressed locally about a point $x \in \widetilde{V} \cap \pi^{-1}(Z(I))$ as $\pi^{*}\left[s_{1}\right]=\widetilde{\sigma}_{\mid \widetilde{V}[f]} h_{1}$, where $\widetilde{\sigma}$ is the generator at $x$ for the stalk of the principal sheaf ideal $\left(\pi^{*}\left[\sigma_{0}\right], \ldots, \pi^{*}\left[\sigma_{L}\right]\right)$, and $\left\{h_{1}=0\right\}$ defines with $\widetilde{\sigma}$ on $\widetilde{V}[f]$ (about $x$ ) a complete intersection. Then it follows from the case studied at the beginning that the current

$$
T[f]\left[s_{1}\right]_{\mid Z(I)}=\left[\left[\bar{\partial}\left(\frac{\left|s_{1}\right|^{2 \lambda}}{s_{1}}\right) \wedge T[f]\right]_{\lambda=0}\right]_{\mid Z(I)}
$$

is annihilated by $I$. Suppose that $l \geq 2$ and that we know that $T\left[s_{1}, \ldots, s_{l-1}\right]$ is annihilated by $I^{l-1}$, once $s_{1}, \ldots, s_{l-1}$ have been conveniently chosen. Then one has

$$
I^{l-1} T[f]\left[s_{1}, s_{2}, \ldots, s_{l-1}\right]_{\mid Z(I)}=0,
$$

which implies that if $h \in I^{l-1}$, the current $h T[f]\left[s_{1}, s_{2}, \ldots, s_{l-1}\right]_{\mid U \backslash Z(I)}=$ $h T[f]\left[s_{1}, s_{2}, \ldots, s_{l-1}\right]$ is a Coleff-Herrera current. Repeating the argument used for $l=1$, with $T[f]$ replaced by $h T[f]\left[s_{1}, \ldots, s_{l-1}\right]_{\mid U \backslash Z(I)}$, one can choose $s_{l}$ in a generic way (genericity depends here on the previous choices of $s_{1}, \ldots, s_{l-1}$, but is independent of $h$ ), so that, for any $h \in I^{l-1}$,

$$
h T[f]\left[s_{1}, \ldots, s_{l}\right]_{\mid Z(I)}=\left[\left[\bar{\partial}\left(\frac{\left|s_{l}\right|^{2 \lambda}}{s_{l}}\right) \wedge h T\left[f, s_{1}, \ldots, s_{l-1}\right]_{\mid U \backslash Z(I)}\right]_{\lambda=0}\right]_{\mid Z(I)}
$$

is annihilated by $I$. It follows then that, with this convenient choice of $s_{l}$, the current $\left(T[f]\left[s_{1}, \ldots, s_{l}\right]\right)_{\mid Z(I)}$ is annihilated by $I^{l}$. That concludes the proof.

It is natural to call the sequence $\left(\left\langle u^{1}, \sigma\right\rangle, \ldots,\left\langle u^{n-M}, \sigma\right\rangle\right)$ a Vogel sequence for $I$ with respect to the Coleff-Herrera current $T[f]$, if such a sequence is constructed (in a generic way in terms of the $u^{j}$ ) through the inductive procedure described above step-by-step. The corresponding current $\bigwedge_{1}^{n-M} \bar{\partial}\left(1 /\left\langle u^{j}, \sigma\right\rangle\right) \wedge T[f]$ is then called a Vogel residue current (for I with respect to $T[f])$.

Remark 5. When $[V]$ is a purely dimensional cycle in $\mathcal{X}$ (here assimilated to its associated integration current), and $\mathcal{Z} \subset \mathcal{X}$ is a complex submanifold, it is proved in [14], and reinterpreted in algebraic terms in [24], that the (multi) cycle of intersection $[\mathrm{V}] \bullet Z$, defined via the Vogel procedure from a prescribed Vogel sequence, is such that its Chow ideal $\mathcal{I}^{\text {Chow }}([V] \bullet[\mathcal{Z}])$ (see [24] for the definition of this notion) lies in the integral closure of the sheaf of ideals $\left(\mathcal{I}([V]), \mathcal{I}_{\mathcal{Z}}\right)$. Briançon-Skoda's theorem then implies that $\left(\mathcal{I}^{\text {Chow }}([V] \bullet[\mathcal{Z}])\right)^{n} \subset\left(\mathcal{I}([V]), \mathcal{I}_{\mathcal{Z}}\right)$. Proposition 3 can thus be understood as 
an analog result, when $[V]$ is replaced by the Coleff-Herrera $T[f]$, and intersection theory transposed at the (algebraic) level of residue currents instead of the (geometric) one of integration currents.

\subsection{Averaging Vogel residue currents}

Let $\mathcal{X}$ be a $n$-dimensional complex manifold, $U$ be an open set in $\mathcal{X}, M \leq n$, $T$ be a $(0, M) E$-valued Coleff-Herrera current in $U$ (or $\mathcal{T}$ a $(0, M) E$-valued Coleff-Herrera current in $U$ with poles), with respect to a closed analytic subset $V \subset U$ of pure codimension $M$. Let $\mathcal{I}$ be an ideal sheaf in $\mathcal{O}_{\mathcal{X}}(U)$. Assume that $\sigma_{0}, \ldots, \sigma_{L}$ generate $\mathcal{I}(U)$ globally in $U$. One can think of $\left(\sigma_{0}, \ldots, \sigma_{L}\right)$ as an holomorphic section of the trivial bundle $E_{\sigma}=U \times \mathbb{C}^{L+1}$, equipped with its standard hermitian metric. It is natural to propose as an averaged current

$$
\int_{\left(\mathbb{P}^{L}(\mathbb{C})\right)^{m}}\left[\left(\bigwedge_{j=1}^{m} \bar{\partial}\left(\frac{1}{\left\langle u^{j}, \sigma\right\rangle}\right) \wedge(T \text { or } \mathcal{T})\right] d \omega_{L}\left(u^{1}\right) \otimes \cdots \otimes d \omega_{L}\left(u^{m}\right)\right.
$$

(where $m=\min (L+1, n-M+1), \omega_{L}$ denotes the Fubini metric on $\mathbb{P}^{L}(\mathbb{C}$ ) and $\langle u, \sigma\rangle=\sum_{0}^{L} u_{l} \sigma_{l}$ for $\left.u=\left[u_{0}: \cdots: u_{L}\right] \in \mathbb{P}^{L}(\mathbb{C})\right)$, the current obtained as the value at 0 of the $\bigoplus_{r=0}^{n-M} \mathcal{D}^{(0, M+r)}\left(\mathcal{X}, \bigwedge^{r} E_{\sigma}^{*} \otimes E\right)$ current-valued map

$$
\lambda \longmapsto\left(1-\|\sigma\|^{2 \lambda}+\bar{\partial}\|\sigma\|^{2 \lambda} \wedge\left(\sum_{r=1}^{\operatorname{dim} V} \frac{1}{(2 i \pi)^{r}} \frac{\sigma^{*} \wedge\left(\bar{\partial} \sigma^{*}\right)^{r-1}}{\|\sigma\|^{2 r}}\right)\right) \wedge(T \text { or } \mathcal{T}) .
$$

Here $\sigma^{*}=\bar{\sigma}=\sum_{0}^{L} \bar{\sigma}_{l} \otimes e_{l}^{*}$ and $d \sigma=\sum_{0}^{L} d \sigma_{l} \otimes e_{l}$ (if $\left(e_{0}, \ldots, e_{L}\right)$ denotes a standard base of sections for $E_{\sigma}=U \times \mathbb{C}^{L+1}$ ). Because of the local structure theorems for sections of the Coleff-Herrera sheaves (see [32]), this map is holomorphic in some half-plane $\operatorname{Re} \lambda>-\eta$ for some $\eta>0$. It was indeed proved in [8], as a consequence of Crofton's formula, that, if $m=\min (L+$ $1, \operatorname{dim} V+1)$, the averaging

$$
\int_{\left(\mathbb{P}^{L}(\mathbb{C})\right)^{m}}\left[\left(\bigwedge_{j=1}^{m} \bar{\partial}\left(\frac{1}{\left\langle u^{j}, \sigma\right\rangle}\right) \wedge[V]\right) \wedge \bigwedge_{j=1}^{m} d\left\langle u^{j}, \sigma\right\rangle\right] d \omega_{L}\left(u^{1}\right) \otimes \cdots \otimes d \omega_{L}\left(u^{m}\right)
$$

leads to the current

$$
\begin{aligned}
& M_{V}^{\sigma}:= \\
& {\left[\left(1-\|\sigma\|^{2 \lambda}+\bar{\partial}\|\sigma\|^{2 \lambda} \wedge\left(\sum_{r=1}^{\operatorname{dim} V} \frac{1}{(2 i \pi)^{r}} \frac{\sigma^{*} \wedge\left(\bar{\partial} \sigma^{*}\right)^{r-1}}{\|\sigma\|^{2 r}}\right)\right) \wedge[V]\right]_{\lambda=0}(d \sigma, \ldots, d \sigma) .}
\end{aligned}
$$


The action on $(d \sigma, \ldots, d \sigma)$ corresponds here to the contraction operation between the $\bigwedge^{r} E_{\sigma}^{*}$ and $E_{\sigma}$ as follows :

$$
\left(\Phi_{1}^{*} \wedge \cdots \wedge \Phi_{r}^{*}\right)(d \sigma, \ldots, d \sigma)=\frac{\bigwedge_{j=1}^{r} \Phi_{j}^{*}(d \sigma)}{r !}, r=1, \ldots, \operatorname{dim} V
$$

for any $\Phi_{j}^{*}$ of $E_{\sigma}^{*}$-valued currents. The current $(23)$ is $d$-closed and positive in this case. Even though it is not an integration current, the vector of the Lelong numbers $\left(e_{0}(x), \ldots, e_{n-M}(x)\right)$ of its various components (from type $(0,0)$ till type $(n-M, n-M)$ ), at any point $x \in V \cap Z(\mathcal{I})$, coincides with the vector of Segre numbers (see [22]) at $x$ for $\mathcal{I}_{x}$ with respect to $V$, that is the minimum (respect to lexicographic order) of all u-plets $\left(\operatorname{deg}_{x} \gamma_{x, 0}, \ldots, \operatorname{deg}_{x} \gamma_{x, n-M}\right)$, where $\gamma$ is a Vogel cycle at $x$ and $\gamma_{x, k}, k=0, \ldots, n-M$, denotes its component at $x$ with codimension $k$ in $V$ (as a subcycle of $V_{x}$ ). Moreover, the relevant part in the Siu decomposition of the averaged current $M^{\sigma}$ is expressed as

$$
M_{V, \text { relevant }}^{\sigma}=\sum_{k=0}^{\operatorname{dim} V} \sum_{\iota} \beta_{\iota}^{k}\left[Z_{\iota}^{k}\right],
$$

where the $Z_{\iota}^{k}$ are the distinguished varieties of $\mathcal{I}$ with codimension $k$ (distinguished varieties being defined as the images of the irreducible components of the exceptional divisor $\mathcal{E}$ in the normalized blowup of $U$ along the coherent ideal sheaf $\mathcal{I}$ ). Here the $\beta_{\iota}^{k}$ are positive integer coefficients. This generalized version of H. King's formula is proved in [8]. This shows that, at the semi global level, averaging Coleff-Herrera currents, once they are conveniently multiplied in order to become intersection currents, is an operation that fits well with improper intersection theory, such as implemented in [30, 31]. Note that the Segre numbers remain unchanged if one replaces $s=\left(\sigma_{0}, \ldots, \sigma_{L}\right)$ by some u-plet $\left(\tilde{\sigma}_{0}, \ldots, \tilde{\sigma}_{\tilde{L}}\right)$ which generate in $\mathcal{O}_{\mathcal{X}}(U)$ an ideal with, locally about each point, the same integral closure than $\mathcal{I}$. This will emphasizes the role of a current $M^{\sigma}$ (or, to be more precise, residue currents involved in its factorization) with respect to Briançon-Skoda's type theorems.

In order to extend these ideas to the global setting, one assumes that the coherent sheaf $\mathcal{I}$ is globally generated in $\mathcal{X}$ by holomorphic sections $\sigma_{0}, \ldots, \sigma_{L}$ of the same line bundle $\mathcal{O}_{\mathcal{X}}(\Delta)$. Referring to Example 1, the holomorphic sec-

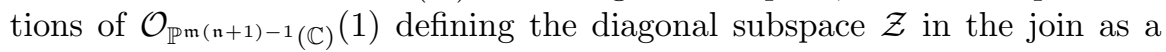
complete intersection, provide a useful illustration of such a situation, the coherent sheaf $\mathcal{I}$ being in this case the radical sheaf $\mathcal{I}_{\mathcal{Z}}$. Let $E_{\sigma}=\bigoplus^{L+1} \mathcal{O}_{\mathcal{X}}(\Delta)$. Choose a metric $\mid$ on $\mathcal{O}_{\mathcal{X}}(\Delta)$, that induces the metric \|\|$=|| \oplus \cdots \oplus||$ on $E_{\sigma}$. Let $E$ be a finite rank holomorphic bundle over $\mathcal{X}$. When $T($ resp. $\mathcal{T})$ is an element in $\mathrm{CH}_{\mathcal{X}, V}(\mathcal{X}, E)$ (resp. in $\mathrm{CH}_{\mathcal{X}, V}(\mathcal{X} ; \star S, E)$ ), it is natural to propose, as the averaged current (21), the current obtained as the value at 0 of the $\bigoplus_{r=0}^{n-M}{ }^{\prime} \mathcal{D}^{(0, M+r)}\left(\mathcal{X}, \bigwedge^{r} E_{\sigma}^{*} \otimes E\right)$ current-valued map (22), where $\sigma^{*}$ denotes the conjugate section of $\sigma$ respect to the metric on $E_{\sigma}$. If $\pi: \widetilde{V} \longrightarrow V$ de- 
notes the normalized blowup of the complex space $\left(V,\left(\mathcal{O}_{\mathcal{X}}\right)_{\mid V}\right)$ along the $\mathcal{O}_{V}$ ideal sheaf $\mathcal{I}_{\mid V}$, generated locally by the holomorphic sections $x \mapsto \sigma_{l}(\iota(x))$ $(\iota$ being the embedding $V \subset \mathcal{X}), l=0, \ldots, L$, and $\mathcal{E}$ denotes the exceptional divisor in this normalized blowup, then one can check that this averaging leads, from the point of view of intersection theory: that is, when $T$ or $\mathcal{T}$ are replaced by the $(M, M)$-current $[V]$, to the construction of the Vogel current

$$
\begin{aligned}
& \operatorname{Vog}_{V, \sigma,||}= \\
& {\left[\left(1-\|\sigma\|^{2 \lambda}+\bar{\partial}\|\sigma\|^{2 \lambda} \wedge\left(\sum_{r=1}^{\operatorname{dim} V} \frac{1}{(2 i \pi)^{r}} \frac{\sigma^{*} \wedge\left(\bar{\partial} \sigma^{*}\right)^{r-1}}{\|\sigma\|^{2 r}}\right)\right) \wedge[V]\right]_{\lambda=0}(d \sigma, \ldots, d \sigma)} \\
& =\left[V^{\mathcal{Z}(\mathcal{I})}\right]+\sum_{r=1}^{\operatorname{dim} V} \pi_{*}\left([\mathcal{E}] \wedge\left(c^{1}\left(\mathcal{O}_{\widetilde{V}}(-\mathcal{E}) \otimes \pi^{*}\left[\Delta_{\mid V}\right]\right)\right)^{r-1}\right) \\
& =\sum_{r=0}^{\operatorname{dim} V} \operatorname{Vog}_{(\ldots) ; r} .
\end{aligned}
$$

We denote as $\Delta_{\mid V}$ the line bundle $\mathcal{O}_{V}(\Delta)$. Here $\pi^{*}[\sigma]=e^{[0]} \times \tau$, where $\tau$ is an holomorphic non vanishing u-plet of sections of $\mathcal{O}_{\tilde{V}}(-\mathcal{E}) \otimes \pi^{*}\left[\Delta_{\mid V}\right]$. The metric on $\mathcal{O}_{\widetilde{V}}(-\mathcal{E})$ is defined by $\left|e^{[0]}\right|_{\mathcal{E}}=\|\sigma \circ \pi\|$. It induces a metric on the divisor $\mathcal{O}_{\widetilde{V}}(-\mathcal{E}) \otimes \pi^{*}\left(\left(\mathcal{O}_{\mathcal{X}}(\Delta)\right)_{\mid V}\right)$, so that

$$
d d^{c} \log \left|e^{[0]}\right|_{\mathcal{E}}^{2}=[\mathcal{E}]+c^{1}\left(\mathcal{O}_{\widetilde{V}}(-\mathcal{E})\right)=[\mathcal{E}]+d d^{c} \log \|\tau\|^{2}-\pi^{*}\left(c^{1}\left(\Delta_{\mid V}\right)\right),
$$

that is

$$
\begin{aligned}
& d d^{c} \log \|\tau\|^{2}=c^{1}\left(\pi^{*}\left[\Delta_{\mid V}\right]\right)+c^{1}\left(\mathcal{O}_{\widetilde{V}}(-\mathcal{E})\right) \\
& =c^{1}\left(\mathcal{O}_{\widetilde{V}}(-\mathcal{E}) \otimes \pi^{*}\left[\Delta_{\mid V}\right]\right)
\end{aligned}
$$

The current $\operatorname{Vog}_{(\ldots)}=\sum_{r=0}^{\operatorname{dim} V} \operatorname{Vog}_{(\ldots) ; r}$ (that can also be considered as a current on $\left.\left(V, \mathcal{O}_{V}\right)\right)$ is related to the Segre current

$\operatorname{Seg}_{V, \sigma,||}=\left[V^{\mathcal{Z}(\mathcal{I}) \cap V}\right]+\sum_{r=1}^{\operatorname{dimV}} \pi_{*}\left([\mathcal{E}] \wedge\left(c^{1}\left(\mathcal{O}_{\widetilde{V}}(-\mathcal{E})\right)\right)^{r-1}\right)=\sum_{r=0}^{\operatorname{dimV}} \operatorname{Seg}_{V, \sigma,|| ; r}$

thanks to the algebraic relations

$$
\operatorname{Vog}_{V, \sigma,|| ; r}=\sum_{l=0}^{r-1}\left(\begin{array}{c}
r-1 \\
l
\end{array}\right) \operatorname{Seg}_{V, \sigma,|| ; r-l} \wedge\left(c_{1}\left(\Delta_{\mid V}\right)\right)^{l}, r=1, \ldots, \operatorname{dim} V .
$$

An important particular case occurs where $T$ is a Coleff-Herrera residue of the form $\bigwedge_{j=1}^{M} \bar{\partial}\left(1 / s_{j}\right), s_{1}, \ldots, s_{M}$ being respective holomorphic sections of Cartier divisors $\Delta_{j}, j=1, \ldots, M$, on $\mathcal{X}$, such that the $s_{j}^{-1}(0)$ define a 
complete intersection. In this particular case, one can consider the $(M+L+1)$ holomorphic bundle $E_{\Delta, \sigma}=\bigoplus_{j=1}^{M} \mathcal{O}_{\mathcal{X}}\left(\Delta_{j}\right) \oplus E_{\sigma}$, equipped with the metric $\bigoplus_{1}^{M}||_{j} \oplus\|\|$ (where ||$_{j}$ denotes an hermitian metric on $\mathcal{O}_{\mathcal{X}}\left(\Delta_{j}\right), j=$ $1, \ldots, M)$, and propose as an alternative averaged version for all Vogel residue currents

$\bigwedge_{j=1}^{m} \bar{\partial}\left(\frac{1}{\left\langle u^{j}, \sigma\right\rangle}\right) \wedge \bigwedge_{j=1}^{M} \bar{\partial}\left(\frac{1}{s_{j}}\right), u^{j} \in \mathbb{P}^{L}(\mathbb{C}), 1 \leq j \leq m=\min (L+1, \operatorname{dim} V+1)$

the current obtained (still through the analytic continuation process) as the value at $\lambda=0$ of the $\bigoplus_{r=0}^{n-M}{ }^{\prime} \mathcal{D}^{(0, M+r)}\left(\mathcal{X}, \bigwedge^{r} E_{\Delta, \sigma}^{*}\right)$ current-valued map

$\lambda \longmapsto 1-\|s \oplus \sigma\|^{2 \lambda}+\bar{\partial}\|s \oplus \sigma\|^{2 \lambda} \wedge\left(\sum_{r=1}^{\operatorname{dim} V} \frac{1}{(2 i \pi)^{r}} \frac{(s \oplus \sigma)^{*} \wedge\left(\bar{\partial}(s \oplus \sigma)^{*}\right)^{r-1}}{\|s \oplus \sigma\|^{2 r}}\right)$,

where $s \oplus \sigma=s_{1} \oplus \cdots \oplus s_{M} \oplus \sigma$ and $(s \oplus \sigma)^{*}$ denotes its conjugate section respect to the metric which has been chosen on $E_{\Delta, \sigma}$. The two currents, which belong to $\bigoplus_{r=0}^{n-M}{ }^{\prime} \mathcal{D}^{(0, M+r)}\left(\mathcal{X}, \bigwedge^{r} E_{\Delta, \sigma}^{*}\right)$,

$$
\left[\left(1-\|\sigma\|^{2 \lambda}+\bar{\partial}\|\sigma\|^{2 \lambda} \wedge\left(\sum_{r=1}^{\operatorname{dim} V} \frac{1}{(2 i \pi)^{r}} \frac{\sigma^{*} \wedge\left(\bar{\partial} \sigma^{*}\right)^{r-1}}{\|\sigma\|^{2 r}}\right)\right) \wedge \bigwedge_{j=1}^{M} \bar{\partial}\left(1 / s_{j}\right)\right]_{\lambda=0}
$$

and

$$
\left[1-\|s \oplus \sigma\|^{2 \lambda}+\bar{\partial}\|s \oplus \sigma\|^{2 \lambda} \wedge\left(\sum_{r=1}^{\operatorname{dim} V} \frac{1}{(2 i \pi)^{r}} \frac{(s \oplus \sigma)^{*} \wedge\left(\bar{\partial}(s \oplus \sigma)^{*}\right)^{r-1}}{\|s \oplus \sigma\|^{2 r}}\right)\right]_{\lambda=0}
$$

coincide when $\operatorname{codim}\left(s^{-1}(0) \cap \sigma^{-1}(0)\right)=M+L+1$, see [34], but differ in general. They can both be used (see $[3,7]$ ) to materialize the residual obstruction for the exactness of the generically exact Koszul complex

$$
\begin{aligned}
& 0 \rightarrow \bigwedge^{M+L+1} E_{\Delta, \sigma}^{*} \stackrel{\lrcorner_{s \oplus \sigma}}{\longrightarrow} \bigwedge^{M+L} E_{\Delta, \sigma}^{*} \longrightarrow \cdots \stackrel{\lrcorner_{s \oplus \sigma}}{\longrightarrow} \\
& \bigwedge_{l+1} E_{\Delta, \sigma}^{*} \stackrel{\lrcorner_{s \oplus \sigma}}{\longrightarrow} \bigwedge^{l} E_{\Delta, \sigma}^{*} \longrightarrow \cdots \stackrel{\lrcorner_{s \oplus \sigma}}{\longrightarrow} E_{\Delta, \sigma}^{*} \stackrel{\lrcorner_{s \oplus \sigma}}{\longrightarrow} \mathcal{X} \times \mathbb{C},
\end{aligned}
$$

since they are both annihilated (as currents) by the operator $2 i \pi\rfloor_{s \oplus \sigma}-\bar{\partial}$, where $\rfloor_{s \oplus \sigma}$ denotes the interior multiplication by the holomorphic section $s \oplus \sigma$ of $E_{\Delta, \sigma}$. 


\section{About a result by M. Hickel, M. Andersson and E. Götmark}

In order to emphasize what averaged Vogel residue currents introduced in Section 2 could be useful for, and, in parallel, to illustrate how far using their use reveals to be successful, let us focus on the effective geometric global formulation of Briançon-Skoda's theorem in $\mathbb{C}\left[X_{1}, \ldots, X_{\mathfrak{n}}\right]$, as obtained first by M. Hickel in [20], and then reformulated (and thus reproved), using the frame developed above, by M. Andersson and E. Götmark in [5]. Before stating the result, one needs to recall basic facts about Lojasiewicz exponents at infinity in $\mathbb{C}^{\mathfrak{n}} \subset \mathbb{P}^{\mathfrak{n}}(\mathbb{C})=\mathbb{C}^{\mathfrak{n}} \cup\left\{z_{0}=0\right\}$. Let $p_{1}, \ldots, p_{\mathfrak{m}}$ be $\mathfrak{m}$ polynomials in $\mathbb{C}\left[X_{1}, \ldots, X_{\mathfrak{n}}\right]$, together with their homogenizations $P_{1}, \ldots, P_{\mathfrak{m}}$, considered as respective holomorphic sections of the Cartier divisors $\mathcal{O}_{\mathbb{P}^{\mathfrak{n}}(\mathbb{C})}\left(\operatorname{deg} p_{j}\right)$. Let $\mathcal{E}=\bigcup_{\iota} \mathcal{E}_{\iota}$ be the (reduced) exceptional divisor of the normalized blowup $\widetilde{\mathbb{P}}^{\mathfrak{n}}(\mathbb{C}) \stackrel{\pi}{\longrightarrow} \mathbb{P}^{\mathfrak{n}}(\mathbb{C})$ of $\mathbb{P}^{\mathfrak{n}}(\mathbb{C})$ along the coherent ideal sheaf $\mathcal{I}(P)$, generated locally by the holomorphic sections $P_{1}, \ldots, P_{\mathfrak{m}}$. Let $\mu_{\iota}(P)$ be the multiplicity of $\mathcal{I}(P) \cdot \mathcal{O}_{\widetilde{\mathbb{P}}(\mathbb{C})}$, and $\mu_{\iota}\left(z_{0}\right)$ be the multiplicity of $\mathcal{I}_{\left\{z_{0}=0\right\}} \cdot \mathcal{O}_{\widetilde{\mathbb{P}}(\mathbb{C})}$ along the same component. The Lojaciewicz exponent $\nu_{\infty}(P)$ is defined as

$$
\nu_{\infty}(P)=\sup _{\iota}\left(\frac{\mu_{\iota}(P)}{\mu_{\iota}\left(z_{0}\right)}\right)
$$

In the special case where $\mathfrak{m}=\mathfrak{n}$ and $\operatorname{dim}_{\mathbb{C}^{\mathfrak{n}}} p^{-1}(0) \leq 0$, one has the Lojasiewicz inequality at infinity in $\mathbb{C}^{\mathfrak{n}} \subset \mathbb{P}^{\mathfrak{n}}(\mathbb{C})$, namely

$$
\sum_{j=1}^{\mathfrak{n}} \frac{\left|p_{j}(\zeta)\right|}{|\zeta|^{\operatorname{deg} p_{j}}} \geq \frac{\kappa}{|\zeta|^{\nu_{\infty}(P)}}, \quad|\zeta|>>1
$$

for some strictly positive constant $\kappa$. In particular, for $p$ to be in this case a proper map from $\mathbb{C}^{\mathfrak{n}}$ into $\mathbb{C}^{\mathfrak{n}}$, it is enough that $\nu_{\infty}(P)<\operatorname{deg} p_{j}, j=1, \ldots, \mathfrak{m}$. Notice that, when $\mathfrak{m}=\mathfrak{n}$ and $\operatorname{dim}_{\mathbb{C}^{\mathfrak{n}}} p^{-1}(0) \leq 0$, the current

$$
\left[\bigwedge_{1}^{\mathfrak{n}} \bar{\partial}\left(\frac{1}{P_{j}}\right)\right]_{\mid\left\{z_{0}=0\right\}}
$$

is annihilated by $z_{0}^{\mathfrak{n} \nu_{\infty}(P)}$. This can be seen using (as in the proof of Proposition 3) the normalized blowup of $\mathbb{P}^{\mathfrak{n}}(\mathbb{C})$ along the coherent ideal sheaf $\mathcal{I}_{\left\{z_{0}=0\right\}}$. On the other hand, the current

$$
\left[\bigwedge_{1}^{\mathfrak{n}} \bar{\partial}\left(\frac{1}{P_{j}}\right)\right]_{\mid \mathbb{C}^{n}}
$$


is annihilated by any homogeneous polynomial of the form $Q$ such that the $\operatorname{map} \zeta \mapsto Q(1, \zeta) /\|p(\zeta)\|^{\mathfrak{n}}$ is locally bounded in $\mathbb{C}^{\mathfrak{n}}$ (see [5]).

M. Hickel's result ([20], revisited with the analytic methods presented above in [5]) can be stated as follows (we just mention here the result when $\mathfrak{m} \leq \mathfrak{n})$.

Theorem $4([\mathbf{5}, \mathbf{2 0}])$. Assume $\mathfrak{m} \leq \mathfrak{n}$ and let $p_{1}, \ldots, p_{\mathfrak{m}}$ be $\mathfrak{m}$ polynomials in $\mathbb{C}\left[X_{1}, \ldots, X_{\mathfrak{n}}\right]$. Let $q$ be a polynomial in $\mathbb{C}\left[X_{1}, \ldots, X_{\mathfrak{n}}\right]$ such that the function $|q| /|p|^{\mathfrak{m}}$ is locally bounded in $\mathbb{C}^{\mathfrak{n}}$. Then, there exist polynomials $a_{1}, \ldots, a_{\mathfrak{m}} \in$ $\mathbb{C}\left[X_{1}, \ldots, X_{\mathfrak{n}}\right]$ such that $q \equiv \sum_{j=1}^{\mathfrak{m}} a_{j} p_{j}$ with $\operatorname{deg} a_{j} p_{j} \leq \operatorname{deg} q+\left[\mathfrak{m} \nu_{\infty}(p)\right]+1$, where $[\gamma]$ denotes the integer part of the rational number $\gamma$.

As a consequence of this theorem, it appears that the effective realization of the global Briançon-Skoda theorem ( $q$ being in the ideal $\left(p_{1}, \ldots, p_{\mathfrak{m}}\right)$ in $\mathbb{C}\left[X_{1}, \ldots, X_{\mathfrak{n}}\right]$ if the germ $q_{\zeta}$, for each $\zeta \in p^{-1}(0)$, lies in the $\mathfrak{m}$-power of the integral closure of the ideal $\left(p_{1, \zeta}, \ldots, p_{, \zeta}\right)$ in the local ring $\left.\mathcal{O}_{\mathbb{C}^{\mathrm{n}}, \zeta}\right)$ can be achieved with degree estimates for the quotients $a_{j}$ controlled by the geometric Bézout theorem, which means that the effectivity of the problem is governed by geometric intersection theory.

The proof of Theorem 4 relies on the use of the averaged BochnerMartinelli version $(22)\left(E_{\sigma}=\bigoplus_{1}^{\mathfrak{m}} \mathcal{O}_{\mathbb{P}^{n}(\mathbb{C})}\left(d_{j}\right)\right.$ with the Fubini-Study metric on each component, $\left.\sigma=P, V=\mathbb{P}^{\mathfrak{n}}(\mathbb{C})=\mathcal{X}\right)$, of all currents

$$
\bigwedge_{1}^{m} \bar{\partial}\left(\frac{1}{\left\langle u^{j}, P_{j}\right\rangle}\right)
$$

$u^{1}, \ldots, u^{m} \in \mathbb{P}^{\mathfrak{m}-1}(\mathbb{C}), m=\min (\operatorname{dim}(\{P=0\})+1, \mathfrak{m})$, with respect to the tensorized Fubini Study metric on $\mathbb{P}^{\mathfrak{m}-1}(\mathbb{C}) \otimes \cdots \otimes \mathbb{P}^{\mathfrak{m}-1}(\mathbb{C})$ ( $m$ times). As pointed out in [24], when dealing with intersection problems involving arithmetic aspects, it is more adequate to use a specific Vogel cycle of intersection instead of what could be understood as an averaged version, for example the intersection cycle constructed in [31] and reinterpreted in [8] in the currential setting. The methods presented here (end of Section 1 and subsection 2.2) aim precisely to give some support to the following conjecture :

Conjecture 1 (Global arithmetic Briançon-Skoda) Let $p_{1}, \ldots, p_{\mathfrak{m}}$ be $\mathfrak{m}$ polynomials in $\mathbb{Z}\left[X_{1}, \ldots, X_{\mathfrak{n}}\right]$ with degrees bounded by $d$ and logarithmic sizes bounded by $h$. Let $q \in \mathbb{Z}\left[X_{1}, \ldots, X_{\mathfrak{n}}\right]$ be such the function $q /|p|^{\min (\mathfrak{m}, \mathfrak{n})}$ is locally bounded in $\mathbb{C}^{\mathfrak{n}}$. Then, one can find $\delta \in \mathbb{N}^{*}, a_{1}, \ldots, a_{\mathfrak{m}} \in \mathbb{Z}\left[X_{1}, \ldots, X_{\mathfrak{n}}\right]$ such that

$$
\begin{aligned}
& \delta q^{\kappa(\mathfrak{n})}=\sum_{j=1}^{\mathfrak{m}} a_{j} p_{j}, \quad \max _{j}\left(\operatorname{deg}\left(a_{j} p_{j}\right)\right) \leq \kappa(\mathfrak{n}) \operatorname{deg} q+\kappa_{0}(\mathfrak{n}) d^{\gamma_{0} \min (\mathfrak{n}, \mathfrak{m})} \\
& \max _{j}\left(h(\delta), h\left(a_{j} p_{j}\right)\right) \leq \kappa(\mathfrak{n}) h(q)+\kappa_{1}(\mathfrak{n})(h+\log \mathfrak{m}) d^{\gamma_{1} \min (\mathfrak{n}+1, \mathfrak{m})},
\end{aligned}
$$


where $\kappa(\mathfrak{n}), \kappa_{0}(\mathfrak{n}), \kappa_{1}(\mathfrak{n})$ are numerical constants depending only on the number $\mathfrak{n}$ of variables, $\gamma_{0}, \gamma_{1}$ being universal constants (idealistically $\kappa(\mathfrak{n})=1$, $\left.\kappa_{0}(\mathfrak{n})=\mathfrak{n}, \gamma_{0}=\gamma_{1}=1\right)$.

This result was obtained by M. Elkadi in [16] when $\operatorname{dim}\left(p^{-1}(0)\right)=0$. On the other hand, the arithmetic membership problem can be solved with such bounds (30) when $\mathfrak{m} \leq \mathfrak{n}$ and $\left(p_{1}, \ldots, p_{\mathfrak{m}}\right)$ defines a complete intersection in $\mathbb{C}^{\mathfrak{n}}$ (see [17]), which includes in particular the case of the arithmetic nullstellensatz ([10], [25], [15]). This fits well with the ideas that govern the construction of Vogel sequences. Nevertheless, it is known now that methods based on multidimensional residue calculus (relying essentially on CauchyWeil integral formula and associated Bergman-Weil developments) do not provide the sharpest bounds for the arithmetic nullstellensatz, which is truly a problem related to arithmetic intersection theory (see the recent approach in [15], based on a arithmetic version of O. Perron's theorem used for the algebraic nullstellensatz in [21]). As a consequence, this makes more clear that such methods were in fact more in the spirit of Conjecture 1. Using the Stückrad-Vogel approach [30], combined with a precise analysis of the Vogel residue currents involved (description of their annihilators, explicit computations of the restrictions to $\mathbb{C}^{n}$ of the auxiliary Coleff-Herrera currents involved in their expansion, see Propositions 2 and 3 above, together with the concluding comments in Section 1), seems to be a natural way to tackle such a conjecture. It is indeed necessary to overcome the difficulty which is inherent to the fact that averaging such Vogel residue currents in order to get suitable Bochner-Martinelli currents (for control of the degree in effectivity questions) does not preserve the arithmetic structure of the data (which would be necessary in order to get in parallel control on the heights). It seems also opportune to mention that the initial approach to Theorem 4 by F. Amoroso in [2] relies on the Northcott-Rees notion of superficial elements in ideals, that is also present in the construction of Vogel sequences (more specifically of filtered sequences, see [1]).

This presentation of the Coleff-Herrera machinery, combined in subsection 2.2 with that of the Stückrad-Vogel approach $[30,31,8]$, transposed to the context of residue currents instead of integration currents on cycles, intends to be a modest invitation towards such an approach to effectivity questions in arithmetic polynomial geometry, when they require operational tools related to duality, such as Briançon-Skoda's theorem or multidimensional operational residue calculus.

\section{References}

1. Achilles, R., Rams, S. : Intersection numbers, Segre numbers and generalized Samuel multiplicities. Arch. Math. 77, 391-398 (2001) 
2. Amoroso, F. : On a conjecture of C. Berenstein and A. Yger. In : L. Gonzalez Vega and T. Recio (eds.) Algorithms in algebraic geometry and applications (Santander 1994), pp. 17-28. Progress in Mathematics 143, Birkhäuser, 1996

3. Andersson, M. : Residue currents and ideals of holomorphic functions. Bull. Sci. Math. 128, no. 6, 481-512 (2004)

4. Andersson, M. : Coleff-Herrera currents, duality, and Noetherian operators. Preprint Gothenburg (2009), available at arXiv : 0902.3064

5. Andersson, M., Götmark, E. : Explicit representation of membership in polynomial ideals. Math. Ann. 349, no. 2, 345-365 (2011)

6. Andersson, M., Wulcan, E. : Residue currents with prescribed annihilator ideals. Ann. Sci. École Norm. Sup. 40, 985-1007 (2007)

7. Andersson, M., Wulcan, E. : Decomposition of residue currents. J. Reine Angew Math. 638, 103-118 (2010)

8. Andersson, M., Samuelsson, H., Wulcan, E., Yger, A. : Nonproper intersection theory and positive currents I, local aspects. Preprint (2010), available at arXiv : 1009.2458

9. Berenstein, C. A., Gay, R., Vidras, A., Yger, A.: Residue currents and Bézout identities. Progress in Mathematics 114, Birkhäuser, 1993

10. Berenstein C. A, Yger, A. : Residue Calculus and effective Nullstellensatz. Amer. J. of Math. 121, no. 4, 723-796 (1999)

11. Björk, J. E. : Residues and $\mathcal{D}$-modules. In : O.A. Laudal, Piene R. (eds.) The legacy of Niels Henrik Abel, pp. 605-651, Springer-Verlag, Berlin (2004)

12. Björk, J. E., Samuelsson, H. : Regularizations of residue currents. J. Reine Angew. Math. 649, 33-54 (2010)

13. Coleff, N., Herrera, M. : Les courants résiduels associés à une forme méromorphe. Lecture Notes in Math. 633, Springer-Verlag, Berlin, New-York, 1978

14. Cygan, E. : Intersection theory and separation exponent in complex analytic geometry. Ann. Polon. Math. 69, no. 3, 287-299 (1998)

15. D'Andrea, C., Krick, T., Sombra, M. : Heights of varieties in multiprojective spaces and arithmetic nullstellensatz. Preprint (2011), available at arXiv : 1103.4561

16. Elkadi, M. : Une version effective du théorème de Briançon-Skoda dans le cas algébrique discret. Acta Arithm. 66, 201-220 (1994)

17. Elkadi, M. : Bornes pour les degrés et les hauteurs dans le problème de division. Michigan Math. J. 40, 609-618 (1993)

18. Elkadi, M., Yger, A. : Residue calculus and applications. Publ. Res. Inst. Math. Sci. 43, no. 1, 55-73 (2007)

19. Flenner, H., Carroll, L.O., Vogel, W. : Joins and intersections. Springer Monographs in mathematics, Springer, 1999

20. Hickel, M. : Solution d'une conjecture de C. Berenstein et A. Yger et invariants de contact à l'infini. Ann. Inst. Fourier (Grenoble) 51, no. 3, 707-744 (2001)

21. Jelonek, Z. : On the effective Nullstellensatz. Inventiones math. 162, no. 1, 1-17 (2005)

22. Gaffney, T, Gassler, R. : Segre numbers and hypersurface singularities. J. Algebraic Geometry 8, 695-736 (1999)

23. Griffiths, P., Harris, J. : Principles of Algebraic Geometry, Wiley-Interscience, New York, 1978

24. Kollár, J. : Effective Nullstellensatz for arbitrary ideals. Jour. Eur. Math. Soc. 1, no. 3, 313-337 (1999)

25. Krick, T., Pardo, L. M., Sombra, M. : Sharp estimates for the arithmetic Nullstellensatz. Duke Math. J. 109, no. 3, 521-598 (2001)

26. Massey D. B. : Numerical control over Complex Analytic Singularities. Memoirs of the American Mathematical Society 163, no. 778 (2003)

27. Passare, M., Tsikh, A., Yger, A. : Residue currents of the Bochner-Martinelli type. Publicacions Matematiques 44, 85-117 (2000)

28. Samuelsson, H. : Analytic continuation of residue currents. Ark. Mat. 47, no. 1, $127-$ 141 (2009) 
29. Solomin, J. E. : Le résidu logarithmique dans les intersections non complètes. C. R. Acad. Sci. Paris Sér. A-B 284 no. 17, pp. A1061-A1064 (1977)

30. Stückrad, J., Vogel, W. : An algebraic approach to the intersection theory. Queen's Papers in Pure and Appl. Math. 61, 1-32 (1982)

31. Tworzewski, P. : Intersection theory in complex analytic geometry, Ann. Polon. Math. 62, 177-191 (1995)

32. Vidras A., Yger A. : Coleff-Herrera currents revisited. In : Sabadini I., Struppa D.C. (eds.) The Mathematical Legacy of Leon Ehrenpreis, 1930-2010, pp. ..., Springer (2011)

33. Wiebe, H. : Über homologische Invarianten lokaler Ringe. Math. Ann. 179, 257-274 (1969)

34. Wulcan, E. : Products of residue currents of the Cauchy-Fantappié-Leray type. Ark. Math. 45, 157-178 (2007) 